\title{
Community Structure of Leaf-Litter Ants in a Neotropical Dry Forest: A Biogeographic Approach to Explain Betadiversity
}

\author{
Rogério Silvestre, ${ }^{1}$ Manoel F. Demétrio, ${ }^{2}$ and Jacques H. C. Delabie ${ }^{3}$ \\ ${ }^{1}$ Laboratório de Ecologia de Hymenoptera (HECOLAB), Universidade Federal da Grande Dourados, Rodovia Dourados, Itahum, \\ km 12, Cidade Universitária, 79.804-970 Dourados, MS, Brazil \\ ${ }^{2}$ Programa de Pós-Graduação em Entomologia e Conservação da Biodiversidade, Universidade Federal da Grande Dourados (UFGD), \\ Cidade Universitária, 79.804-970 Dourados, MS, Brazil \\ ${ }^{3}$ Laboratório de Mirmecologia, Centro de Pesquisas do Cacau, Convênio UESC/CEPLAC, 45.651-630 Ilhéus, BA, Brazil
}

Correspondence should be addressed to Rogério Silvestre, rogeriosilvestre@ufgd.edu.br

Received 16 March 2011; Revised 9 May 2011; Accepted 8 June 2011

Academic Editor: Jonathan D. Majer

Copyright (C) 2012 Rogério Silvestre et al. This is an open access article distributed under the Creative Commons Attribution License, which permits unrestricted use, distribution, and reproduction in any medium, provided the original work is properly cited.

\begin{abstract}
This paper describes habitat and geographic correlates of ant diversity in Serra da Bodoquena, a poorly surveyed region of centralwestern Brazil. We discuss leaf-litter ant diversity on a regional scale, with emphasis on the contribution of each of the processes that form the evolutionary basis of contemporary beta diversity. The diversity of leaf-litter ants was assessed from a series of 262 Winkler samples conducted in two microbasins within a deciduous forest domain. A total of 170 litter-dwelling ant species in 45 genera and 11 subfamilies was identified. The data showed that the study areas exhibited different arrangements of ant fauna, with a high turnover in species composition between sites, indicating high beta diversity. Our analysis suggests that the biogeographic history of this tropical dry forest in the centre of South America could explain ant assemblage structure more than competitive dominance. The co-occurrence analysis showed that species co-occur less often than expected by chance in only two of the localities, suggesting that, for most of the species, co-occurrences are random. The assessment of the structure of the diversity of litter-dwelling ants is the first step in understanding the beta diversity patterns in this region of great biogeographic importance.
\end{abstract}

\section{Introduction}

The highly diverse ant fauna of the leaf litter in tropical forests has been the focus of many studies investigating the structure of ecological communities, particularly in the last twenty years [1-3]. Approximately $60 \%$ of the entire ant species that are currently known live in leaf litter, where the ant fauna is especially diverse, taxonomically, morphologically, and ecologically [4-6]. Studies on the biogeography and diversity of Formicidae, as well as the processes affecting their maintenance, can be of great interest for planning effective conservation of the biota at a regional scale. Such studies can also contribute to producing new ecological and taxonomic data, particularly in areas where no previous records exist for the group $[7,8]$.

The Serra da Bodoquena, within the Chacoan subregion, borders the provinces Chaco, Cerrado, Pantanal and Parana Forest $[9,10]$ and is a place with no previous ant records. Prado and Gibbs [11] pointed out that seasonal deciduous forests are remnants of a broader continuous distribution that was present in the past, ranging from north-eastern Brazil to Argentina in the Pleistocene dry period. This currently fragmented structure is the result of the dry, cold climate that caused the retraction of wet forests to riversides and the spread of seasonal forests [12]. Deciduous forests comprise discontinuous patches along fertile valleys and basaltic and calcareous rocks in a matrix of Cerrado on the Brazilian Central Plateau. This matrix, intersected by riparian forests, acts as a connection among dry forests in north-eastern Brazil, east of Minas Gerais and São Paulo States, and forest remnants in Pantanal. The vegetation has some floristic similarities to the Amazon and the Paraguayan Chaco $[13,14]$. 
We investigated whether the leaf-litter ant assemblages at Serra da Bodoquena could be explained by current factors, such as ant dominance and competition, or if the community structure was influenced by its geography, which differs between the northern and southern portions of Park.

Some hypotheses regarding the array of situations found in the region could be tested, assuming that the vegetation properties are also valid for the ant assemblage. Despite the biogeographic relationships of the vegetation, current and evolutionary effects of environmental formations may be reflected in the structure of the ant community. Interspecific competition is usually associated with significant divergence and with the principle of limiting similarity [15]. Although niche differentiation is undoubtedly an important concept, it seems insufficient to wholly determine the high levels of local diversity commonly observed in warm climates $[5,6,16,17]$.

Significant aggregations of assemblages have been associated with the presence of environmental filters [18]. The coexistence of species would be more frequent than expected if randomly organized, because of environmental conditions that act as environment filters, allowing only a narrow spectrum of species to survive. We discuss the possibility that the structure of the leaf-litter ant community in Serra da Bodoquena could be influenced by neighbouring landscapes, as it is situated at the intersection between the Pantanal, Chaco, Cerrado, Brazilian Atlantic Forest, and Amazon Forest biomes. Alternatively, the fauna could be completely different and specific to this Seasonal Deciduous Forest.

The following issues were based on three sets of arguments; namely, (i) if the similarity between the sampling sites is high, the ant fauna of the north and south portions of the dry forest could be derived from the same historic processes and by the same selective ecological pressures and could be driven by a single colonisation process (this argument assumes that all species have an equal probability of colonisation in all sites); (ii) if the north and south portions of the forest have a distinct fauna, this suggests that the geographic basis is important to the formation of the ant assemblages once the different portions attained a distinct physiographic structure. (Therefore, the question is whether the faunistic similarity of ant communities between the northern and southern portions of Serra da Bodoquena is low, blocks are likely to be formed through different colonisation processes;) or (iii) if the samples are dissimilar among sites, a series of distinct ecological, spatial, and temporal situations may have contributed to the formation of leaf-litter ant assemblage in the region, and the surrounding environments influence the faunistic colonisation.

The goal in the present study was thus to identify associating parameters between the community structure of leaf-litter ants and the phytophysionomic matrix within the two distinct land portions in Serra da Bodoquena National Park.

In central-western Brazil, the expansion of agriculture and intensive cattle farming has led to a dramatic loss of forests. Thus, it is likely, this insect diversity has already been affected before it is has been thoroughly evaluated. Therefore, the assessment of the structure of the diversity of leaf-litter ants is the first step in understanding these patterns in this region of great biogeographic importance.

\section{Materials and Methods}

This study was carried out in a seasonal deciduous forest area in Serra da Bodoquena National Park (core coordinates: $21^{\circ} 07^{\prime} 16^{\prime \prime} \mathrm{S} 56^{\circ} 28^{\prime} 55^{\prime \prime} \mathrm{W}$ ). This is the only fully protected Federal Reserve of Mato Grosso do Sul, Brazil. It harbours significant portions of seasonal deciduous and semideciduous forests, transitional areas between Cerrado and Brazilian Atlantic Forest, Cerrado and Tropical Seasonal Deciduous Forest, marshes, rocky fields, and anthropic lands with cattle farms.

The western region of the Bodoquena mountain range is formed by a mosaic of vegetational types; lowlands, including savannas steppe, arborous and gramineous Chaco, plus xeromorphic and mesoxeromorphic forests. To the east, there are many cattle farms within what used to be Cerrado vegetation, to the south, there are soybean farms and islands of semi-deciduous forest, and to the north, there are the Pantanal plains. The island of preserved dry forest areas in this region is the largest of those in the centre of South America.

The Serra da Bodoquena National Park has an area of 77,200 ha, made up of a steep plateau in the west, and comprising two distinct land portions that together cover a $300 \times 50 \mathrm{~km}$ area. The area is preserved because it is a watershed that supplies the drainage basins of the Western region of Brazil [19]. The region divides important water catchments. Salobra River, in the Northern land portion, fuels the Miranda River on Pantanal plains, and Perdido River, in the Southern land portion, fuels the Apa River. Both rivers are tributaries of the Paraguay River, although their respective waters only mix after a thousand kilometres (Figure 1).

The locality is sustained by calcareous rocks of the Corumbá group-Neoproterozoic III. It is characterised by a high rocky massif, with altitudes varying between $200 \mathrm{~m}$ and $770 \mathrm{~m}$ asl. Exposed limestone from the Tamengo formation predominates in this karstic region, where rivers are found within canyons [20,21].

The annual average temperatures of the area vary between $22^{\circ} \mathrm{C}$ and $26^{\circ} \mathrm{C}$. The minimum temperature can be as low as $0^{\circ} \mathrm{C}$. The relative humidity is low and rarely reaches $80 \%$, and rainfall varies between $1300 \mathrm{~mm}$ and $1700 \mathrm{~mm}$ a year. The hot and rainy season occurs between October and April, and the cold and dry season from May to September [22].

The survey was carried out from September 2005 to February 2008, with samples taken in the dry and wet seasons, at 10 selected sites, in eight collecting expeditions (in two expedictions has two sites) along the Bodoquena ridge (Table 1), covering the microbasin of Salobra River in the Northern land portion, including the Kadiwéu Indian Reserve, and the microbasin of Perdido River, in the Southern land portion (Figure 2).

The leaf-litter sampling ant was carried out according to the ALL protocol [2], with a few adaptations due to 


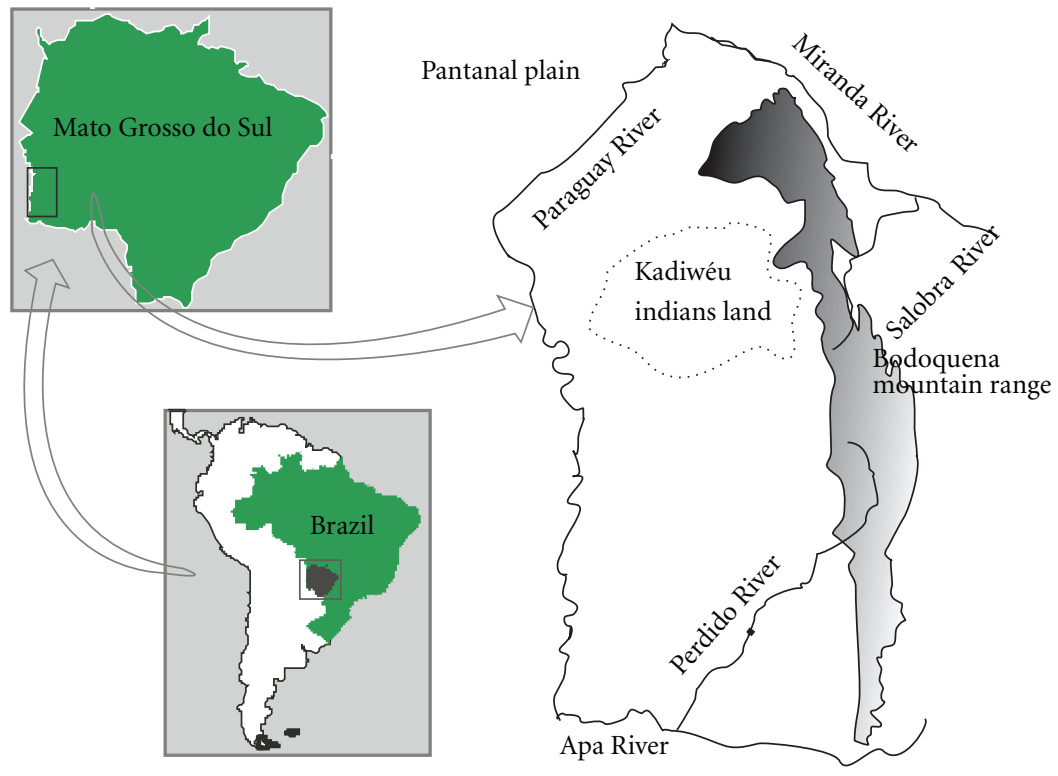

Figure 1: Map of Serra da Bodoquena (Chacoan sub-region), bordering the Pantanal province, State of Mato Grosso do Sul, Brazil.

\section{Sites of capture}

Image chart of study area: Serra da Bodoquena National Park

Polyconic projection-South American datum 1969

Cartographic base: landsat mosaic, path/rows 266/74 + 75;

acquisition dates: 2/Sept/07 + 13May/07

\section{$1: 750000$}

(1) Balneário Perdido River

(2) Sta Maria Farm-Perdido River

(3) Harmonia Farm

(4) Boqueirão Farm

(5) DaMata Farm

(6) Sta Laura Farm and Salobra River-left and right margin

(7) California Farm

(8) Kadiwéu reserve

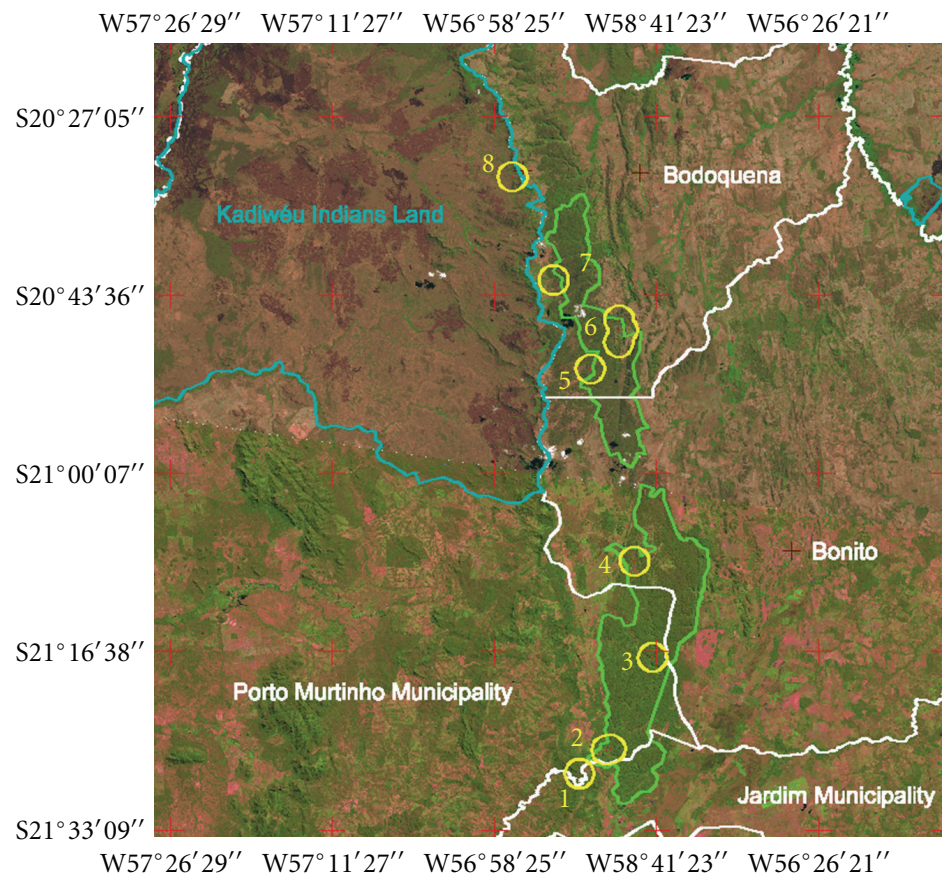

$\mathrm{W} 57^{\circ} 26^{\prime} 29^{\prime \prime} \mathrm{W}^{\prime} 7^{\circ} 11^{\prime} 27^{\prime \prime} \mathrm{W} 56^{\circ} 58^{\prime} 25^{\prime \prime} \mathrm{W} 58^{\circ} 41^{\prime} 23^{\prime \prime}$ W $56^{\circ} 26^{\prime} 21^{\prime \prime}$

FIgURE 2: Landsat image indicating eight localities studied in Serra da Bodoquena National Park, Brazil. Obs: in Salobra river (site 6) three sampling points were performed (III, IV, and IX).

the habitat being composed of limestone rock floors which made it impossible to use pitfall traps in most areas. A total of 262 leaf-litter samples of $1 \mathrm{~m}^{2}$ were extracted using mini-Winkler sacks [23]. A high diversity of microhabitats with a stratified structure was observed in the study areas. Inside the forest, there are calcareous floors and rocks with little-litter accumulation. The sampled points were chosen randomly along each transect of $40 \mathrm{~m} \times 500 \mathrm{~m}$ but set at minimum intervals of $20 \mathrm{~m}$. In each transect a minimum of 25 samples were taken. We searched for microhabitats in dry forest with sufficient leaf-litter accumulation so as to obtain approximate $2 \mathrm{~kg}$. The sample exposure time of the material inside the extractor was 24 hours.

The ant identifications follow Bolton [24, 25], Fernández [26], Baroni-Urbani and De Andrade [27], and LaPolla et al. [28]. Voucher specimens were deposited at the Museu de Biodiversidade da Universidade Federal da Grande Dourados (MuBio-UFGD, Mato Grosso do Sul, Brazil) under the 
TABLE 1: Sampling localities, number of samples per site, altitude above sea level, sampling season, and geographic coordinates.

\begin{tabular}{|c|c|c|c|c|c|}
\hline Points/sites & Number of samples & ASL & Portion & Season & Coordinates \\
\hline \multirow{2}{*}{ (I) Balneário Perdido river } & \multirow{2}{*}{25} & \multirow{2}{*}{$357 \mathrm{~m}$} & \multirow{2}{*}{ South } & \multirow{2}{*}{ Dry } & $21^{\circ} 27^{\prime} 55.00^{\prime \prime} \mathrm{S}$ \\
\hline & & & & & $56^{\circ} 48^{\prime} 34.31^{\prime \prime} \mathrm{W}$ \\
\hline \multirow{2}{*}{ (II) Boqueirão farm } & \multirow{2}{*}{32} & \multirow{2}{*}{$511 \mathrm{~m}$} & \multirow{2}{*}{ South } & \multirow{2}{*}{ Wet } & $21^{\circ} 08^{\prime} 13.94^{\prime \prime} \mathrm{S}$ \\
\hline & & & & & $56^{\circ} 43^{\prime} 28.00^{\prime \prime} \mathrm{W}$ \\
\hline \multirow{2}{*}{ (III) Salobra river-left margin } & \multirow{2}{*}{25} & \multirow{2}{*}{$221 \mathrm{~m}$} & \multirow{2}{*}{ North } & \multirow{2}{*}{ Dry } & $20^{\circ} 46^{\prime} 48.87^{\prime \prime} \mathrm{S}$ \\
\hline & & & & & $56^{\circ} 44^{\prime} 32.78^{\prime \prime} \mathrm{W}$ \\
\hline \multirow{2}{*}{ (IV) Salobra river-right margin } & \multirow{2}{*}{25} & \multirow{2}{*}{$248 \mathrm{~m}$} & \multirow{2}{*}{ North } & \multirow{2}{*}{ Dry } & $20^{\circ} 47^{\prime} 59.94^{\prime \prime} \mathrm{S}$ \\
\hline & & & & & $56^{\circ} 44^{\prime} 54.05^{\prime} \mathrm{W}$ \\
\hline \multirow{2}{*}{ (V) Harmonia farm-Perdido river } & \multirow{2}{*}{25} & \multirow{2}{*}{$460 \mathrm{~m}$} & \multirow{2}{*}{ South } & \multirow{2}{*}{ Wet } & $21^{\circ} 17^{\prime} 09.8^{\prime} \mathrm{S}$ \\
\hline & & & & & $56^{\circ} 41^{\prime} 45.5^{\prime} \mathrm{W}$ \\
\hline \multirow{2}{*}{ (VI) Califórnia farm } & \multirow{2}{*}{25} & \multirow{2}{*}{$464 \mathrm{~m}$} & \multirow{2}{*}{ North } & \multirow{2}{*}{ Wet } & $20^{\circ} 42^{\prime} 11.81^{\prime \prime} \mathrm{S}$ \\
\hline & & & & & $56^{\circ} 50^{\prime} 57.56^{\prime \prime} \mathrm{W}$ \\
\hline \multirow{2}{*}{ (VII) Kadiweu reserve } & \multirow{2}{*}{25} & \multirow{2}{*}{$306 \mathrm{~m}$} & \multirow{2}{*}{ North } & \multirow{2}{*}{ Wet } & $20^{\circ} 32^{\prime} 41.48^{\prime \prime} \mathrm{S}$ \\
\hline & & & & & $56^{\circ} 54^{\prime} 44.66^{\prime \prime} \mathrm{W}$ \\
\hline \multirow{2}{*}{ (VIII) Da Mata farm } & 25 & $578 \mathrm{~m}$ & North & Dry & $20^{\circ} 50^{\prime} 26.16^{\prime \prime} \mathrm{S}$ \\
\hline & & & & & $56^{\circ} 47^{\prime} 31.85^{\prime \prime} \mathrm{W}$ \\
\hline (IX) Sta Laura farm-Salobra river & 30 & $233 \mathrm{~m}$ & North & Wet & $20^{\circ} 45^{\prime} 53.6^{\prime \prime} \mathrm{S}$ \\
\hline & & & & & $56^{\circ} 44^{\prime} 53.11^{\prime \prime} \mathrm{W}$ \\
\hline (X) Sta Maria farm-Perdido river & 25 & $402 \mathrm{~m}$ & South & Wet & $21^{\circ} 25^{\prime} 39.24^{\prime} \mathrm{S}$ \\
\hline & & & & & $56^{\circ} 45^{\prime} 48.90^{\prime \prime} \mathrm{W}$ \\
\hline
\end{tabular}

reference numbers Hym00108F to Hym02332F, at the Laboratório de Mirmecologia, Cocoa Research Centre, (CPDC, Ilhéus, Bahia, Brazil), and at the Museu de Zoologia da Universidade de São Paulo (MZ-USP, São Paulo, Brazil).

The data were considered for sites independently and grouped by land portions, South (presumably under Atlantic Forest influence- Paraná subregion), and North (presumably under Pantanal influence- Amazonian subregion).

The data analysis was based on the species occurrence in samples (frequency), as accepted for quantifying social insects [29]. To estimate species richness, the Chao 2 and Jackknife 2nd-order estimators were calculated usingEstimateS 7.5 [30], which are widely used in ant diversity studies [31-33].

Rarefaction curves showing the expected species richness versus species occurrence were used to assess the sampling efficiency for each sample area [34]. From the observed species richness per site, we estimated the number of species remaining to be sampled using the second-order Jackknife estimator (incidence based). The expected number of species was plotted against number of species records on the $x$-axis (individual-based accumulation curve). This plot provides a measure of species diversity which is robust to sample size effects.

In order to verify if there are differences in betadiversity increasing between northern and southern land portions, the two data sets were compared following a north-south axis and were plotted by increasing ant diversity against the distance between successive sample series in the eight localities.

To analyse site similarity, we used a principal coordinate analysis (PCO) using the Bray-Curtis dissimilarity index $[35,36]$. The similarity among the ant assemblages at the different seasons and altitude was assessed using a cluster analysis (Jaccard coefficient of similarity). The resulting similarity matrix was analysed through a sequential, agglomerative, hierarchical, and nested clustering algorithm, described by Sneath and Sokal [37]. The option used was the Unweighted Pair-Group Method, arithmetic average (UPGMA). This analysis was conducted using the MVSP 3.1 software [38].

The diversity and similarity analyses were run using EstimateS 7.5 [30]. Similarity and distance matrices (Euclidian distance) were compared using a Mantel's test [39]. The data set was analysed using $\mathrm{R}$ software [40], using the Vegan package [41]. The graphic design was constructed with Statistica for Windows 6 [42]. The Morisita-Horn index was used too to evaluate the similarity among the localities, pairwise, because this index is not affected by the number of samples or the species richness, except for very small sampling niches $[43,44]$.

We used EcoSim (version 7.72) to compute random matrices of species co-occurrences [45] to determine whether the mean and variance C-score among samples is larger or smaller than expected by chance. Co-occurrences based on averages that were calculated across all possible pairs of species were randomised (5,000 repetitions) within the constraint of fixed marginal totals, which is an appropriate null model for detecting patterns caused by species interactions [46].

\section{Results}

More than 20,000 ants were captured in the seasonal deciduous forest. We recorded 170 species from 45 genera and 


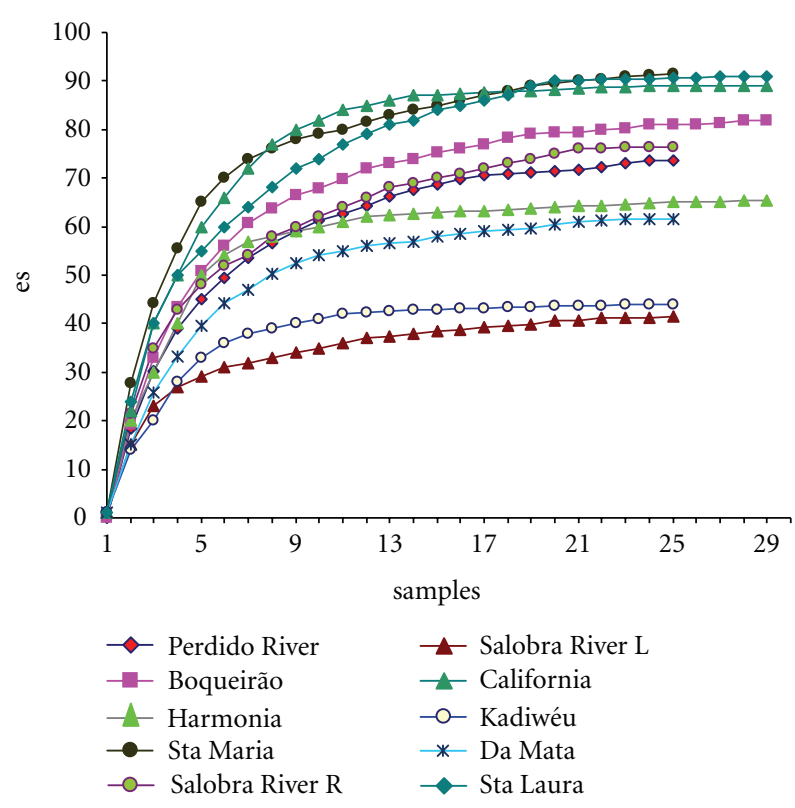

Figure 3: Comparison of incidence-based ant species rarefactions curves showing expected species richness (es) versus sampling effort in ten sampling sites (samples) in the Bodoquena mountain range.

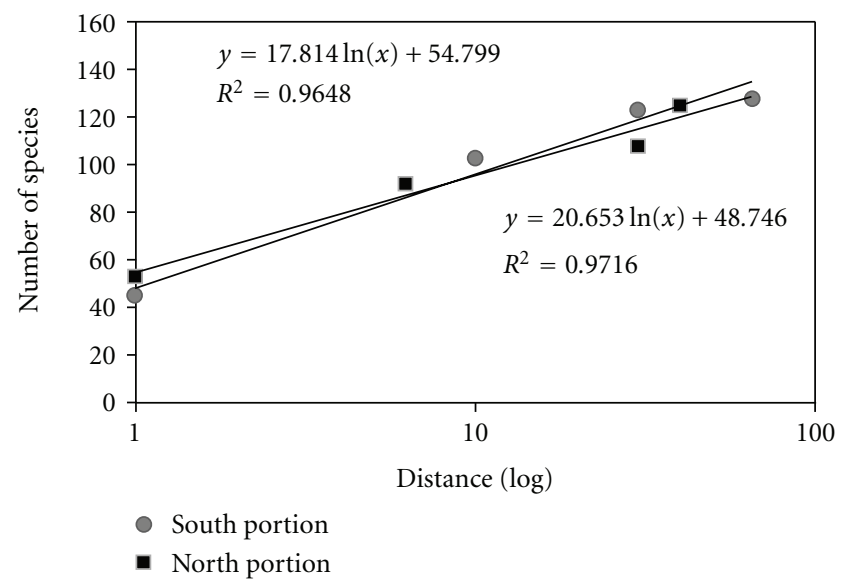

Figure 4: Ant species accumulation (logarithmic scale) in northern and southern portions in Serra da Bodoquena, Mato Grosso do Sul, Brazil. Obs: eight localities were considered, as collecting points III, IV, and IX were grouped as a single sample.

11 subfamilies out of the 15 subfamilies of Formicidae known to occur in the Neotropical Region (Table 2).

The most frequently observed species were Solenopsis (Diplorhoptrum) sp. 1 (91 occurrences), Cyphomyrmex (gr. rimosus) sp. 1 (85), Solenopsis sp. 2 (81), and Hypoponera sp. 7 (77). The most speciose genera were: Hypoponera (21 species), Pheidole (17), Cyphomyrmex (12), Strumigenys (13), Solenopsis (11), and Basiceros (9). We recorded the first observations of the genus Cryptomyrmex in the centralwest Brazilian region. Three new species were found in deciduous forest: Asphinctanilloides sp. new, Amblyopone sp. new, and Probolomyrmex sp. new.

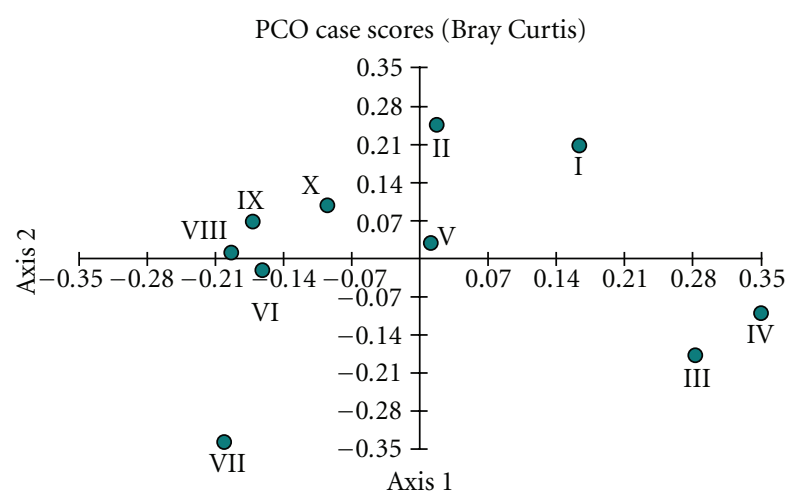

FIGURE 5: PCO analysis of the sites located in the northern and southern portions of Serra da Bodoquena using the Bray-Curtis dissimilarity index (eigenvalues: 0001).

$$
\begin{gathered}
\text { Mantel test } \\
t=-0.0133 p=0.4947
\end{gathered}
$$

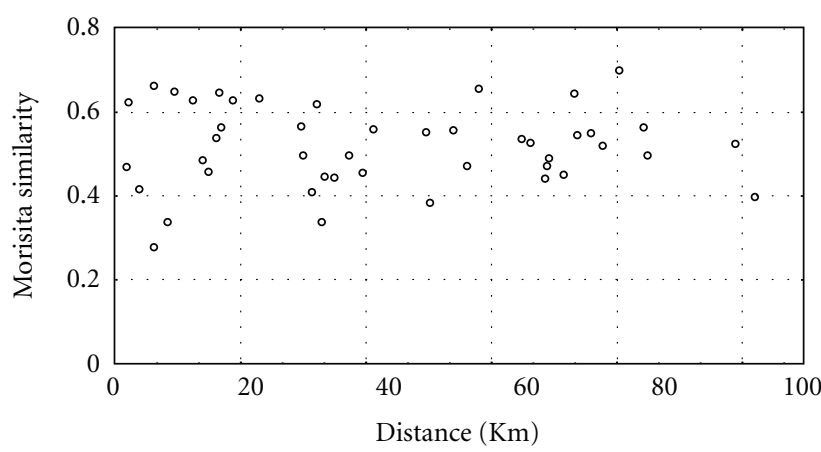

FIGURE 6: Mantel's test analysis between the values obtained using the Morisita-Horn similarity index and the distance between sites $(\mathrm{km})$.

A total of 37 species were recorded only once (singletons). The total number of singletons represents approximately $20 \%$ of all ant species collected in the present study (Table 3 ).

Rarefaction curves (Figure 3 ) show the sampling effort in each sample site, for each land portion. Evidence of asymptotes indicates that most leaf litter ant species were sampled with the number of samples used.

The comparison of northern and southern data sets did not reveal any difference in the betadiversity of the ant communities. The two distributions follow more or less the same pattern of species substitution in relation to increasing the distance of sample sites (Figure 4).

The PCO analysis (Figure 5) shows a consistency of groups between northern and southern land portions. The analysis grouped areas of southern (I, II, and V) and also grouped the sites of northern (VI, VIII, and IX); the site X (southern) is close with this group. The samples most similar were taken in the Salobra River (III and IV) in the same season (dry).

There was no correlation between the similarity (Morisita-Horn) among species frequencies in the communities 
TAble 2: Records of occurrences of the 170 species collected at 10 sampling sites in Serra da Bodoquena National Park, Mato Grosso do Sul, Brazil.

\begin{tabular}{|c|c|c|c|c|c|c|c|c|c|c|}
\hline Species & $\mathrm{I}$ & II & III & IV & $\mathrm{V}$ & VI & VII & VIII & IX & $\mathrm{X}$ \\
\hline \multicolumn{11}{|l|}{ Amblyoponinae } \\
\hline \multicolumn{11}{|l|}{ Tribe Amblyoponini } \\
\hline Amblyopone elongata (Santschi 1912) & - & - & - & - & 2 & - & - & - & - & - \\
\hline Amblyopone lurilabes Lattke 1991 & - & 5 & - & - & - & - & 1 & - & - & 一 \\
\hline Amblyopone sp. new & 1 & - & - & - & - & - & - & - & - & - \\
\hline \multicolumn{11}{|l|}{ Cerapachyinae } \\
\hline \multicolumn{11}{|l|}{ Tribe Cerapachyini } \\
\hline Cerapachys splendens Borgmeier 1957 & - & - & - & - & - & 1 & - & - & - & 1 \\
\hline \multicolumn{11}{|l|}{ Dolichoderinae } \\
\hline \multicolumn{11}{|l|}{ Tribe Dolichoderini } \\
\hline Azteca alfari Emery 1893 & 4 & 3 & 2 & 1 & 4 & 3 & - & 1 & 4 & 一 \\
\hline Dolichoderus sp. 1 & 1 & - & - & - & - & - & - & - & - & - \\
\hline Dorymyrmex sp. 1 & 1 & - & - & - & - & - & - & - & - & - \\
\hline Linepithema humile (Mayr 1868) & 2 & - & 1 & - & 4 & - & - & - & - & - \\
\hline \multicolumn{11}{|l|}{ Ecitoninae } \\
\hline \multicolumn{11}{|l|}{ Tribe Ecitonini } \\
\hline Neivamyrmex sp. 1 & 一 & - & 2 & - & - & 2 & - & 1 & 2 & 2 \\
\hline Neivamyrmex sp. 2 & - & 1 & - & - & - & - & - & - & - & 一 \\
\hline \multicolumn{11}{|l|}{ Ectatomminae } \\
\hline \multicolumn{11}{|l|}{ Tribe Ectatommini } \\
\hline Ectatomma brunneum Smith 1858 & - & - & - & - & - & - & - & - & - & 1 \\
\hline Ectatomma edentatum Roger 1863 & - & - & - & - & - & - & - & - & 1 & - \\
\hline Gnamptogenys striatula Mayr 1884 & 一 & - & - & - & 3 & - & - & - & 2 & 2 \\
\hline Gnamptogenys (gr. striatula) sp. 1 & 一 & - & 1 & - & - & - & - & - & - & 1 \\
\hline Gnamptogenys sulcata (Smith 1858) & - & - & - & - & - & - & - & - & 1 & 一 \\
\hline \multicolumn{11}{|l|}{ Tribe Typhlomyrmecini } \\
\hline Typhlomyrmex rogenhoferi Mayr 1862 & 一 & - & 1 & - & - & - & - & - & - & - \\
\hline Typhlomyrmex sp. 1 & - & - & - & 1 & - & - & - & - & - & 一 \\
\hline \multicolumn{11}{|l|}{ Formicinae } \\
\hline \multicolumn{11}{|l|}{ Tribe Camponotini } \\
\hline Camponotus crassus Mayr 1862 & - & - & - & - & - & - & - & - & 1 & - \\
\hline Camponotus sp. 1 & - & - & - & - & - & 1 & - & - & - & - \\
\hline Camponotus sp. 2 & - & - & - & - & - & - & - & - & - & 1 \\
\hline \multicolumn{11}{|l|}{ Tribe Plagiolepidini } \\
\hline Brachymyrmex sp. 1 & 4 & 3 & 1 & 2 & - & 3 & 1 & - & - & 3 \\
\hline Brachymyrmex sp. 2 & 7 & - & 4 & - & - & - & - & 3 & - & - \\
\hline Brachymyrmex sp. 3 & 1 & 1 & - & - & - & - & - & - & 2 & - \\
\hline Brachymyrmex sp. 4 & 一 & - & - & - & - & - & - & 3 & - & - \\
\hline Nylanderia fulva (Mayr 1862) & - & 1 & - & - & 3 & 2 & - & 1 & - & 1 \\
\hline Nylanderia sp. 1 & - & - & 2 & - & 1 & 1 & 3 & 1 & - & - \\
\hline Nylanderia sp. 2 & 1 & - & 1 & - & 3 & 1 & - & 3 & 1 & 1 \\
\hline Nylanderia sp. 3 & - & - & - & - & - & - & 2 & - & - & 2 \\
\hline Nylanderia sp. 4 & - & - & - & - & - & 1 & - & - & - & 一 \\
\hline Nylanderia sp. 5 & - & - & - & - & - & 1 & - & - & - & - \\
\hline Paratrechina longicornis (Latreille 1802) & 1 & 3 & 一 & 2 & 3 & 一 & - & 2 & 4 & 1 \\
\hline
\end{tabular}


Table 2: Continued.

\begin{tabular}{|c|c|c|c|c|c|c|c|c|c|c|}
\hline Species & I & II & III & IV & $\mathrm{V}$ & VI & VII & VIII & IX & $\mathrm{X}$ \\
\hline \multicolumn{11}{|l|}{ Leptanilloidinae } \\
\hline Asphinctanilloides sp. new & - & - & - & - & - & 1 & - & - & - & - \\
\hline \multicolumn{11}{|l|}{ Myrmicinae } \\
\hline \multicolumn{11}{|l|}{ Tribe Adelomyrmecini } \\
\hline Cryptomyrmex boltoni (Fernández 2003) & - & - & - & - & 1 & - & - & - & - & - \\
\hline \multicolumn{11}{|l|}{ Tribe Attini } \\
\hline Acromyrmex subterraneus (Forel 1893) & - & 3 & - & - & - & - & - & - & - & - \\
\hline Acromyrmex sp. 1 & 2 & - & - & - & - & - & - & - & - & - \\
\hline Acromyrmex sp. 2 & - & 4 & - & - & - & - & - & - & - & - \\
\hline Apterostigma manni Weber 1938 & - & 1 & - & - & - & - & - & - & - & - \\
\hline Apterostigma pilosum Mayr 1865 & 1 & 1 & - & - & - & - & - & - & - & - \\
\hline Apterostigma wasmanni Forel 1892 & 4 & 1 & - & - & - & - & - & - & - & 9 \\
\hline Atta sp. 1 & 2 & - & - & - & - & - & - & - & - & - \\
\hline Cyphomyrmex lectus (Forel 1911) & - & - & 5 & - & - & 一 & - & - & - & - \\
\hline Cyphomyrmex olitor Forel 1893 & - & 1 & - & - & - & 一 & - & - & - & - \\
\hline Cyphomyrmex (gr. rimosus) sp. 1 & 11 & 9 & 7 & 4 & 6 & 13 & 8 & 3 & 10 & 14 \\
\hline Cyphomyrmex (gr. rimosus) sp. 2 & 6 & 8 & 5 & - & 8 & 4 & - & 6 & 7 & 6 \\
\hline Cyphomyrmex (gr. rimosus) sp. 3 & - & 2 & - & - & - & 6 & - & 1 & 4 & 5 \\
\hline Cyphomyrmex (gr. rimosus) sp. 4 & - & 1 & - & - & 2 & 1 & - & - & - & - \\
\hline Cyphomyrmex (gr. rimosus) sp. 5 & - & 2 & - & - & - & 1 & - & - & - & 5 \\
\hline Cyphomyrmex (gr. rimosus) sp. 6 & - & - & - & - & - & 2 & - & - & - & - \\
\hline Cyphomyrmex (gr. rimosus) sp. 7 & - & 1 & - & - & - & 2 & - & - & 3 & - \\
\hline Cyphomyrmex (gr. rimosus) sp. 8 & - & 2 & - & - & - & - & - & 2 & 2 & - \\
\hline Cyphomyrmex (gr. strigatus) sp. 1 & - & - & - & - & 8 & 一 & - & - & - & - \\
\hline Cyphomyrmex (gr. strigatus) sp. 2 & 一 & - & - & - & - & - & - & - & - & 3 \\
\hline Mycocepurus goeldii (Forel 1893) & - & 1 & 12 & 11 & 3 & 5 & 5 & 2 & 6 & 6 \\
\hline Mycocepurus smithii (Forel 1893) & - & 1 & - & - & - & - & - & - & - & - \\
\hline Mycocepurus sp. 1 & 1 & - & - & - & - & - & - & - & - & - \\
\hline Myrmicocrypta sp. 1 & - & 3 & - & 1 & 1 & - & - & - & - & - \\
\hline Sericomyrmex (gr. amabilis) sp. 1 & - & - & 3 & 2 & - & 2 & - & 2 & - & - \\
\hline Sericomyrmex (gr. amabilis) sp. 2 & - & - & - & - & - & - & - & 1 & - & - \\
\hline Sericomyrmex sp. 1 & - & 4 & - & - & - & 3 & - & - & - & - \\
\hline Trachymyrmex sp. 1 & 4 & - & - & - & - & - & - & - & - & - \\
\hline Trachymyrmex sp. 2 & 1 & 1 & - & - & - & - & - & - & - & - \\
\hline \multicolumn{11}{|l|}{ Tribe Blepharidattini } \\
\hline Wasmannia auropunctata (Roger 1863) & - & 2 & 3 & 2 & - & 9 & - & 3 & - & 1 \\
\hline Wasmannia lutzi Forel 1908 & 1 & 2 & 1 & - & - & - & - & 4 & 1 & 4 \\
\hline Wasmannia sp. 1 & - & - & - & - & 3 & 4 & 5 & 1 & - & 1 \\
\hline Wasmannia sp. 2 & - & - & - & - & - & 2 & - & 3 & 1 & 4 \\
\hline Wasmannia sp. 3 & - & - & - & - & - & - & - & 1 & 2 & - \\
\hline \multicolumn{11}{|l|}{ Tribe Cephalotini } \\
\hline Cephalotes atratus (Linnaeus 1758) & 1 & - & - & - & 一 & - & - & - & - & - \\
\hline Cephalotes sp. 1 & - & - & - & - & - & - & - & - & 1 & 1 \\
\hline Cephalotes sp. 2 & - & - & - & - & - & - & - & 1 & 1 & - \\
\hline Procryptocerus alternatus Smith 1876 & 1 & - & - & - & - & - & - & - & - & - \\
\hline \multicolumn{11}{|l|}{ Tribe Crematogastrini } \\
\hline Crematogaster curvispinosa Mayr 1862 & - & - & 4 & - & - & 1 & 1 & - & - & - \\
\hline Crematogaster sp. 1 & - & - & 3 & - & 2 & - & - & - & - & - \\
\hline
\end{tabular}


TABle 2: Continued.

\begin{tabular}{|c|c|c|c|c|c|c|c|c|c|c|}
\hline Species & $\mathrm{I}$ & II & III & IV & $\mathrm{V}$ & $\mathrm{VI}$ & VII & VIII & IX & $\mathrm{X}$ \\
\hline Crematogaster sp. 2 & 1 & - & - & 2 & 4 & - & 1 & - & - & - \\
\hline Crematogaster sp. 3 & - & 2 & - & - & - & - & - & - & - & - \\
\hline Crematogaster sp. 4 & - & 3 & - & - & - & - & - & - & - & - \\
\hline \multicolumn{11}{|l|}{ Tribe Dacetini } \\
\hline Basiceros disciger (Mayr 1887) & - & - & - & 一 & 1 & - & - & - & - & - \\
\hline B. stenognathum (Brown \& Kempf 1960) & 4 & 9 & - & 13 & 11 & 11 & 4 & - & 8 & 7 \\
\hline Basiceros (Octostruma) balzani (Emery 1894) & 7 & - & 10 & 14 & 12 & 10 & - & - & 7 & 3 \\
\hline Basiceros (Octostruma) simoni (Emery 1887) & - & - & - & - & 7 & 7 & - & - & 7 & 4 \\
\hline Basiceros (Octostruma) rugifera (Mayr 1887) & - & - & - & - & 6 & 6 & 6 & - & 3 & 6 \\
\hline Basiceros (Octostruma) sp. 1 & - & - & - & - & 5 & - & - & 4 & 2 & 4 \\
\hline Basiceros (Octostruma) sp. 2 & - & - & - & - & 2 & - & - & - & 2 & - \\
\hline Basiceros (Octostruma) sp. 3 & - & 5 & - & 一 & - & - & - & - & - & - \\
\hline Basiceros (Octostruma) sp. 4 & 4 & - & - & - & - & 2 & - & - & - & - \\
\hline Strumigenys eggersi Emery 1890 & 3 & 3 & 7 & - & 12 & 9 & 5 & 3 & 16 & 4 \\
\hline Strumigenys (gr. elongata) sp. 1 & - & - & 2 & - & - & 3 & - & - & - & - \\
\hline Strumigenys xenochelyna (Bolton 2000) & 3 & 4 & - & - & 14 & - & - & - & - & - \\
\hline Strumigenys sp. 1 & - & - & - & 7 & 2 & - & - & - & - & 2 \\
\hline Strumigenys sp. 2 & - & 2 & - & - & 2 & 4 & - & - & 2 & 4 \\
\hline Strumigenys sp. 3 & - & - & - & - & - & - & 3 & - & - & - \\
\hline Strumigenys sp. 4 & 1 & - & - & - & - & 一 & - & - & - & 一 \\
\hline Strumigenys sp. 5 & 3 & 6 & - & - & 8 & - & - & - & - & - \\
\hline Strumigenys sp. 6 & - & 5 & 9 & 2 & 5 & 4 & 6 & 1 & 14 & - \\
\hline Strumigenys sp. 7 & - & - & 1 & - & - & 2 & - & - & 1 & - \\
\hline Strumigenys sp. 8 & - & - & 4 & - & - & - & - & - & 2 & - \\
\hline Strumigenys sp. 9 & - & - & 1 & - & 2 & 3 & - & - & - & 2 \\
\hline Strumigenys sp. 10 & - & - & - & - & - & - & 1 & - & - & - \\
\hline \multicolumn{11}{|l|}{ Tribe Myrmicini } \\
\hline Hylomyrma balzani (Emery 1894) & - & - & - & - & 1 & - & - & - & - & - \\
\hline Hylomyrma sp. 1 & - & - & 2 & - & - & - & - & - & - & - \\
\hline \multicolumn{11}{|l|}{ Tribe Pheidolini } \\
\hline Pheidole (gr. flavens) sp. 1 & - & - & - & 一 & - & - & - & - & - & 1 \\
\hline Pheidole gertrudae Forel 1886 & - & - & - & 1 & - & - & - & - & 1 & 2 \\
\hline Pheidole sp. 1 & 4 & 3 & 2 & 2 & 6 & 7 & 5 & 5 & 8 & 4 \\
\hline Pheidole sp. 2 & 2 & 11 & 3 & 2 & 5 & 5 & 7 & 5 & - & 3 \\
\hline Pheidole sp. 3 & - & 4 & 2 & 13 & 8 & - & - & 4 & 14 & 3 \\
\hline Pheidole sp. 4 & 1 & 3 & 1 & 4 & 7 & - & - & - & 4 & 3 \\
\hline Pheidole sp. 5 & 2 & - & 2 & - & 5 & - & - & - & 11 & 2 \\
\hline Pheidole sp. 6 & 2 & - & 1 & - & - & - & - & - & 3 & 2 \\
\hline Pheidole sp. 7 & - & - & 2 & 2 & 2 & - & - & - & - & - \\
\hline Pheidole sp. 8 & - & - & - & - & - & - & - & - & 1 & - \\
\hline Pheidole sp. 9 & - & - & - & - & 1 & 2 & 2 & - & 2 & - \\
\hline Pheidole sp. 10 & - & - & 2 & 3 & - & - & - & - & 1 & - \\
\hline Pheidole sp. 11 & - & - & - & - & - & 3 & 3 & - & - & - \\
\hline Pheidole sp. 12 & - & - & - & - & - & 1 & 3 & - & - & - \\
\hline Pheidole sp. 13 & - & - & - & - & - & 4 & 2 & - & - & - \\
\hline Pheidole sp. 14 & - & - & - & - & - & - & - & 1 & - & - \\
\hline Pheidole sp. 15 & - & - & - & - & - & 9 & 6 & - & 1 & - \\
\hline
\end{tabular}


TABle 2: Continued.

\begin{tabular}{|c|c|c|c|c|c|c|c|c|c|c|}
\hline Species & I & II & III & IV & $\mathrm{V}$ & VI & VII & VIII & IX & $\mathrm{X}$ \\
\hline \multicolumn{11}{|l|}{ Tribe Solenopsidini } \\
\hline Carebara sp. 1 & 8 & 10 & 6 & 3 & 6 & 9 & 2 & 3 & 9 & 3 \\
\hline Carebara sp. 2 & 2 & 3 & 1 & - & 7 & 4 & 6 & - & 8 & 2 \\
\hline Megalomyrmex silvestrii Wheeler 1909 & - & - & - & - & - & 3 & - & 3 & - & - \\
\hline Megalomyrmex wallacei Mann 1916 & - & - & - & - & 一 & - & 3 & - & - & - \\
\hline Monomorium sp. 1 & - & - & 14 & 2 & - & - & - & - & - & - \\
\hline Oxуероесиs sp. 1 & - & - & 1 & - & - & - & - & - & - & - \\
\hline Solenopsis (gr. geminata) sp. 1 & - & - & 1 & - & - & - & - & - & - & - \\
\hline Solenopsis (gr. invicta) sp. 1 & 4 & 2 & - & - & - & 4 & - & 1 & 3 & 14 \\
\hline Solenopsis (gr. invicta) sp. 2 & 1 & - & - & - & - & - & - & - & 1 & - \\
\hline Solenopsis (Diphorhoptrum) sp. 1 & 10 & 8 & 6 & 10 & 10 & 12 & 5 & 8 & 7 & 15 \\
\hline Solenopsis sp. 2 & 5 & 6 & 4 & 2 & 3 & 9 & - & 22 & 20 & 10 \\
\hline Solenopsis sp. 3 & 3 & 2 & 5 & 4 & 2 & 4 & 9 & 3 & 2 & 4 \\
\hline Solenopsis sp. 4 & 8 & 6 & 8 & 9 & 一 & 4 & - & 3 & 1 & 2 \\
\hline Solenopsis sp. 5 & 1 & - & - & - & - & 4 & - & - & 5 & 4 \\
\hline Solenopsis sp. 6 & - & - & - & - & - & 9 & - & 3 & 3 & 3 \\
\hline Solenopsis sp. 7 & 2 & 6 & - & - & - & 4 & - & 4 & 4 & 1 \\
\hline Solenopsis sp. 8 & - & - & - & - & - & - & - & - & 10 & - \\
\hline \multicolumn{11}{|l|}{ Tribe Stenammini } \\
\hline Rogeria alzatei Kugler 1994 & - & - & - & - & - & - & 1 & - & - & - \\
\hline Rogeria lirata Kugler 1994 & - & 1 & - & - & - & 1 & 7 & - & - & - \\
\hline Rogeria sp. 1 & - & - & 1 & - & - & - & - & - & 2 & - \\
\hline Rogeria sp. 2 & - & - & 1 & - & - & - & - & - & 2 & - \\
\hline \multicolumn{11}{|l|}{ Ponerinae } \\
\hline \multicolumn{11}{|l|}{ Tribe Ponerini } \\
\hline Anochetus diegensis Forel 1912 & 2 & - & 7 & 4 & 3 & 5 & 6 & 2 & 9 & 10 \\
\hline Hypoponera sp. 1 & 10 & 11 & 8 & 11 & 15 & 6 & - & - & - & 8 \\
\hline Hypoponera sp. 2 & 4 & 13 & 9 & 9 & 7 & - & 4 & - & 7 & 9 \\
\hline Hypoponera sp. 3 & - & - & 7 & 6 & - & 17 & 6 & 3 & - & 4 \\
\hline Hypoponera sp. 4 & - & 6 & - & - & 3 & 8 & 2 & - & 7 & 4 \\
\hline Hypoponera sp. 5 & - & 1 & - & - & 4 & - & - & - & - & 6 \\
\hline Hyроропеra sp. 6 & 2 & 3 & - & - & - & 18 & 7 & 8 & 11 & 11 \\
\hline Hypoponera sp. 7 & 4 & 5 & - & - & 13 & 6 & 13 & 12 & 17 & 7 \\
\hline Hypoponera sp. 8 & - & 9 & - & - & 2 & 3 & - & - & 3 & 6 \\
\hline Hypoponera sp. 9 & 5 & 2 & - & - & - & - & - & - & 2 & 4 \\
\hline Hypoponera sp. 10 & - & 3 & - & - & 6 & 1 & 3 & 1 & 9 & 2 \\
\hline Hypoponera sp. 11 & - & - & - & - & - & - & - & - & 1 & 1 \\
\hline Hypoponera sp. 12 & - & - & - & - & - & - & - & - & - & 1 \\
\hline Hypoponera sp. 13 & - & - & - & - & - & - & - & - & - & 4 \\
\hline Hypoponera sp. 14 & - & - & - & - & - & 6 & - & - & - & 2 \\
\hline Hypoponera sp. 15 & - & - & - & - & 一 & 1 & - & - & - & 1 \\
\hline Hypoponera sp. 16 & - & - & - & - & 3 & - & - & - & - & - \\
\hline Hypoponera sp. 17 & - & - & - & - & - & - & - & - & - & 2 \\
\hline Hypoponera sp. 18 & - & - & - & - & 1 & 3 & - & 2 & - & - \\
\hline Hypoponera sp. 19 & - & 2 & - & - & - & 2 & - & 3 & - & - \\
\hline Hypoponera sp. 20 & - & 1 & - & - & - & 1 & - & - & - & - \\
\hline Hypoponera sp. 21 & - & 6 & - & - & 一 & 4 & - & - & - & - \\
\hline
\end{tabular}


TABle 2: Continued.

\begin{tabular}{|c|c|c|c|c|c|c|c|c|c|c|}
\hline Species & I & II & III & IV & $\mathrm{V}$ & VI & VII & VIII & IX & $\mathrm{X}$ \\
\hline Leptogenys sp. 1 & - & 1 & 1 & - & 1 & - & - & - & - & - \\
\hline Odontomachus bauri Emery 1892 & - & - & 2 & - & - & 2 & - & - & - & - \\
\hline Odontomachus chelifer (Latreille 1802) & - & - & - & - & - & 2 & - & - & - & - \\
\hline Odontomachus meinerti Forel 1905 & 2 & - & 4 & - & 2 & 2 & 1 & - & 3 & 1 \\
\hline Pachycondyla harpax (Fabricius 1804) & - & - & - & - & - & - & - & - & 4 & - \\
\hline Pachycondyla lunaris (Emery 1896) & - & - & - & - & - & - & - & - & - & 1 \\
\hline Pachycondyla marginata (Roger 1861) & - & - & - & - & - & - & - & - & 1 & - \\
\hline Pachycondyla ferruginea (Smith 1858) & - & - & 12 & 2 & - & - & - & 1 & - & - \\
\hline \multicolumn{11}{|l|}{ Proceratiinae } \\
\hline \multicolumn{11}{|l|}{ Tribe Probolomyrmecini } \\
\hline Probolomyrmex sp. new & - & - & - & 4 & - & - & - & - & 1 & 7 \\
\hline Probolomyrmex petiolatus Weber 1940 & - & - & 1 & - & - & - & - & - & - & - \\
\hline \multicolumn{11}{|l|}{ Pseudomyrmecinae } \\
\hline \multicolumn{11}{|l|}{ Tribe Pseudomyrmecini } \\
\hline Pseudomyrmex gracilis (Fabricius 1804) & 2 & - & - & - & - & - & - & - & - & 3 \\
\hline
\end{tabular}

TABLE 3: Species richness, estimated richness (Chao 2, Jackknife 2), diversity index, and the number of "singletons" and "doubletons".

\begin{tabular}{|c|c|c|c|c|c|c|}
\hline Sites & Number of observed species & (Chao 2) & Jackknife $2^{\mathrm{a}}$. order & Shannon-Wiener index & Singletons & Doubletons \\
\hline I & 52 & 65.14 & 74.26 & 3.67 & 17 & 11 \\
\hline II & 62 & 73.64 & 82.52 & 3.85 & 16 & 11 \\
\hline III & 54 & 67.14 & 76.26 & 3.66 & 17 & 11 \\
\hline IV & 33 & 33.67 & 29.94 & 3.18 & 4 & 12 \\
\hline $\mathrm{V}$ & 57 & 59.91 & 62.29 & 3.79 & 8 & 11 \\
\hline VI & 72 & 80.65 & 88.80 & 3.98 & 15 & 13 \\
\hline VII & 36 & 39.60 & 42.87 & 3.39 & 6 & 5 \\
\hline VIII & 45 & 61.33 & 66.03 & 3.45 & 14 & 6 \\
\hline IX & 69 & 79.80 & 89.68 & 3.86 & 18 & 15 \\
\hline $\mathrm{X}$ & 70 & 82.04 & 91.38 & 3.96 & 17 & 12 \\
\hline Total & 170 & 231.70 & 250.38 & 4.40 & 37 & 17 \\
\hline
\end{tabular}

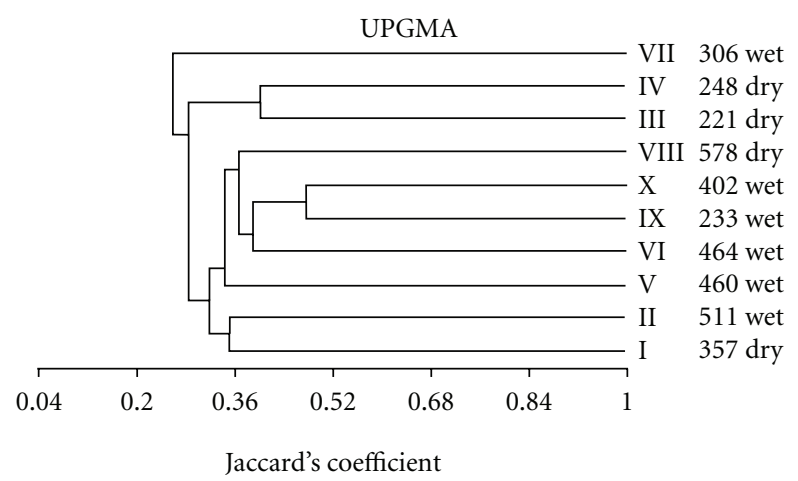

Figure 7: Dendrogram of the similarities (Jaccard coefficient, UPGMA cluster analysis) among 10 sampling sites in the Bodoquena mountain range to leaf-litter ant's fauna. Wet and dry mean seasons, and numbers mean altitude (als).

and the geographical distance $(\mathrm{km})$ between the sites $(r=$ $-0.002 ; P=0.49)$ (Table 4; Figure 6).
The similarity was compared between areas in relation to season and altitude. The samples made during wet season were richer in species, and samples performed in same seasons (VI, IX, and X) appeared grouped. There was no consistent pattern between the assemblages according the altitude $(r=0.214 ; P=0.55)$ (Figure 7).

The co-occurrence analysis indicated that species cooccurred less often than expected by chance in only two of the localities sampled $P$ (observed $\geq$ expected $)=0.003$, for the Balneário Perdido river, and $P$ (observed $\geq$ expected $)=$ 0.042 for the Santa Maria Perdido River, both in the southern micro-basin, suggesting that species co-occurrences are random (Figure 8).

\section{Discussion}

Our results suggest that the studied sites exhibit different arrangements of ant fauna with a replacement in abundant species across sampling units, which results in high beta diversity. Gotelli and Ellison [47] suggested that speciesenergy relationships, in addition to other factors that are 
TABLE 4: Similarity analysis, richness, species shared and distance between sample sites in a pairwise comparison of the leaf-litter ant assemblages from Serra da Bodoquena, Brazil.

\begin{tabular}{|c|c|c|c|c|c|}
\hline \multicolumn{2}{|c|}{ Localities } & \multirow{2}{*}{$\begin{array}{c}\text { Shared species } \\
30\end{array}$} & \multirow{2}{*}{$\frac{\text { Chao shared (estimator) }}{36.14}$} & \multirow{2}{*}{$\frac{\text { Morisita-Horn }}{0.67}$} & \multirow{2}{*}{$\begin{array}{c}\text { Distance }(\mathrm{Km}) \\
30.05\end{array}$} \\
\hline Balneário & Boqueirão & & & & \\
\hline & Salobra left margin & 25 & 30.02 & 0.54 & 76 \\
\hline & Salobra right margin & 18 & 21.14 & 0.54 & 73.77 \\
\hline & Harmonia & 26 & 29.18 & 0.63 & 23 \\
\hline & Califórnia & 25 & 25.83 & 0.56 & 84.4 \\
\hline & Kadiweu reserve & 16 & 16.59 & 0.39 & 102 \\
\hline & Da Mata & 20 & 23.02 & 0.47 & 69 \\
\hline & Sta Laura & 30 & 36.44 & 0.51 & 77.73 \\
\hline & Sta Maria & 32 & 35.76 & 0.66 & 6.2 \\
\hline \multirow[t]{8}{*}{ Boqueirão } & Salobra left & 22 & 24.69 & 0.45 & 39.5 \\
\hline & Salobra right & 20 & 21.49 & 0.49 & 37.3 \\
\hline & Harmonia & 31 & 33.99 & 0.64 & 16.7 \\
\hline & Califórnia & 36 & 40.73 & 0.55 & 49.7 \\
\hline & Kadiweu reserve & 18 & 19.14 & 0.44 & 68.6 \\
\hline & Da Mata & 26 & 30.15 & 0.44 & 33.5 \\
\hline & Sta Laura & 32 & 34.93 & 0.55 & 41.3 \\
\hline & Sta Maria & 35 & 39.71 & 0.61 & 32.2 \\
\hline \multirow[t]{7}{*}{ Salobra left } & Salobra right & 25 & 26.48 & 0.62 & 2.25 \\
\hline & Harmonia & 28 & 32.67 & 0.47 & 56.13 \\
\hline & Califórnia & 30 & 34.48 & 0.48 & 13.96 \\
\hline & Kadiweu reserve & 17 & 18.28 & 0.40 & 31.46 \\
\hline & Da Mata & 24 & 26.26 & 0.33 & 8.43 \\
\hline & Sta Laura & 29 & 38.81 & 0.46 & 1.81 \\
\hline & Sta Maria & 29 & 37.35 & 0.45 & 71.5 \\
\hline \multirow[t]{6}{*}{ Salobra right } & Harmonia & 22 & 24.07 & 0.55 & 54 \\
\hline & Califórnia & 19 & 19.22 & 0.45 & 14.95 \\
\hline & Kadiweu reserve & 14 & 14.23 & 0.33 & 33 \\
\hline & Da Mata & 18 & 20.67 & 0.27 & 6.28 \\
\hline & Sta Laura & 20 & 23.23 & 0.41 & 3.88 \\
\hline & Sta Maria & 23 & 24.24 & 0.49 & 69.3 \\
\hline \multirow[t]{5}{*}{ Harmonia } & Califórnia & 33 & 35.42 & 0.52 & 66.34 \\
\hline & Kadiweu reserve & 22 & 22.82 & 0.49 & 85 \\
\hline & Da Mata & 23 & 28.73 & 0.38 & 50.3 \\
\hline & Sta Laura & 33 & 33.54 & 0.65 & 58 \\
\hline & Sta Maria & 36 & 37.78 & 0.56 & 17 \\
\hline \multirow[t]{4}{*}{ Califórnia } & Kadiweu reserve & 29 & 31.70 & 0.62 & 18.84 \\
\hline & Da Mata & 33 & 37.57 & 0.53 & 16.26 \\
\hline & Sta Laura & 37 & 40.34 & 0.62 & 12.5 \\
\hline & Sta Maria & 43 & 48.18 & 0.69 & 80.5 \\
\hline \multirow[t]{3}{*}{ Kadiweu } & Da Mata & 16 & 18.11 & 0.44 & 35 \\
\hline & Sta Laura & 20 & 20.42 & 0.56 & 29.7 \\
\hline & Sta Maria & 22 & 22.97 & 0.52 & 98.9 \\
\hline \multirow[t]{2}{*}{ Da Mata } & Sta Laura & 30 & 39.10 & 0.64 & 9.56 \\
\hline & Sta Maria & 30 & 33.74 & 0.53 & 64.9 \\
\hline Sta Laura & Sta Maria & 45 & 52.68 & 0.64 & 73.22 \\
\hline
\end{tabular}


Balneario Perdido River

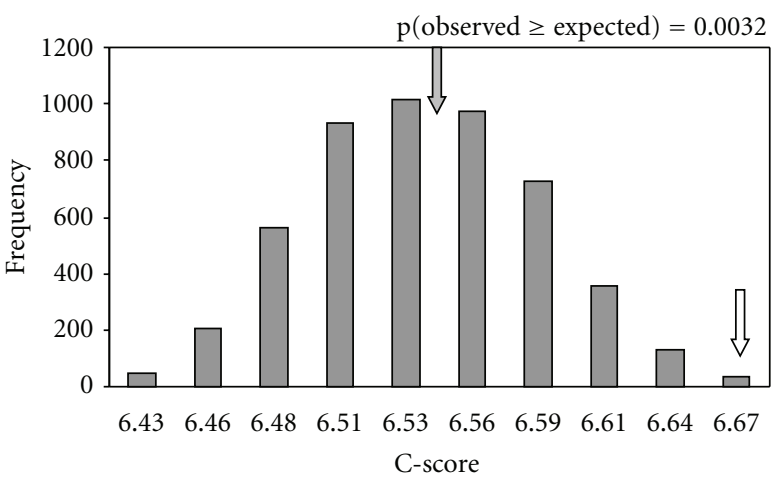

(a)

Sta Laura Farm I

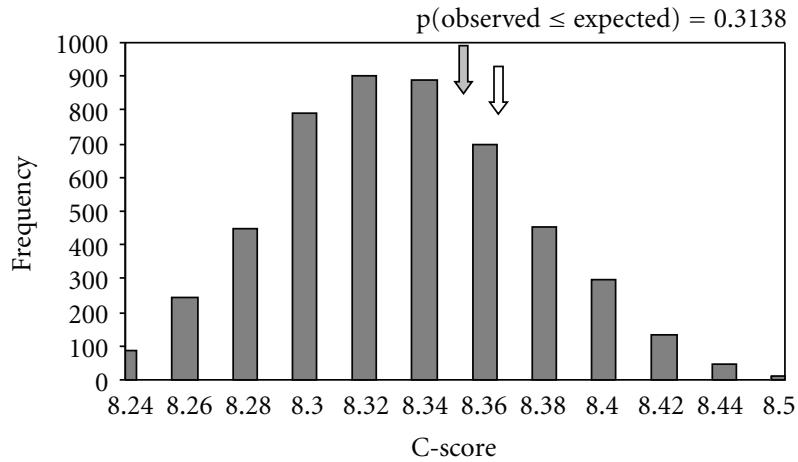

(c)

Harmonia Farm-Perdido River

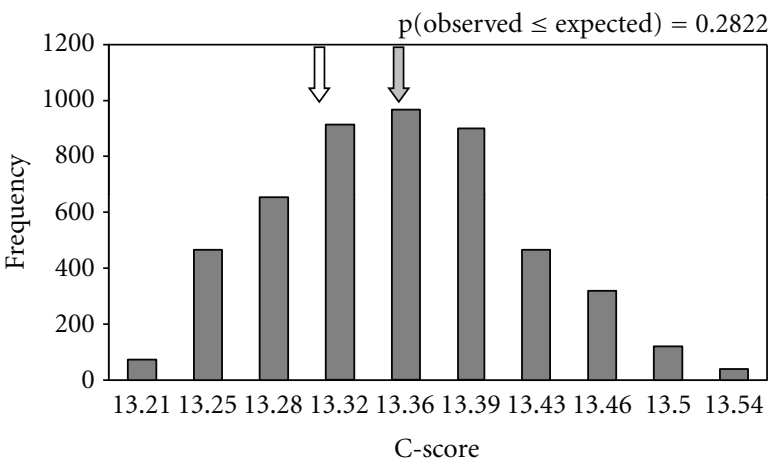

(e)

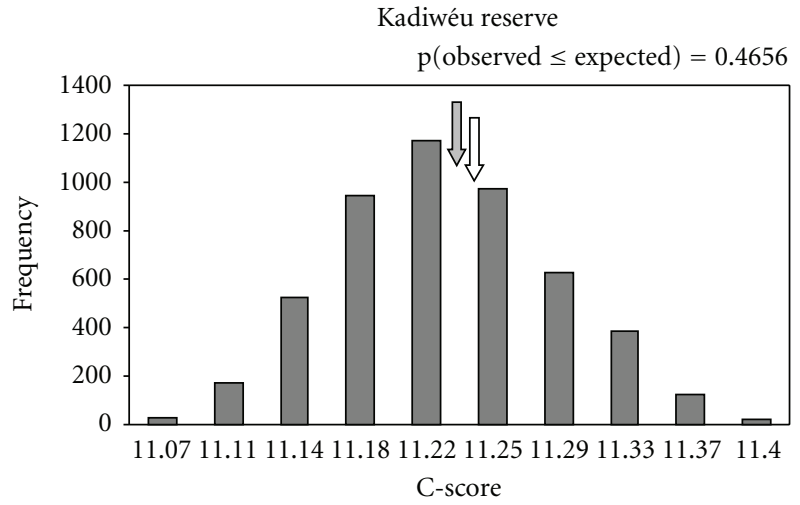

(g)

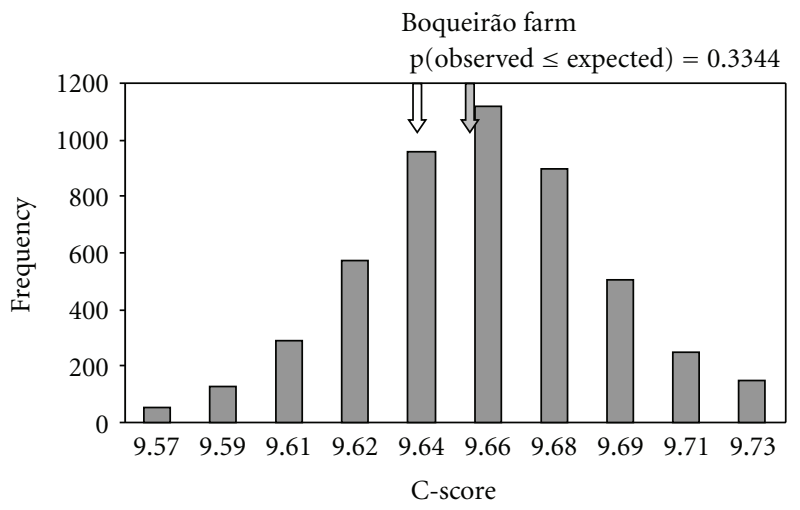

(b)

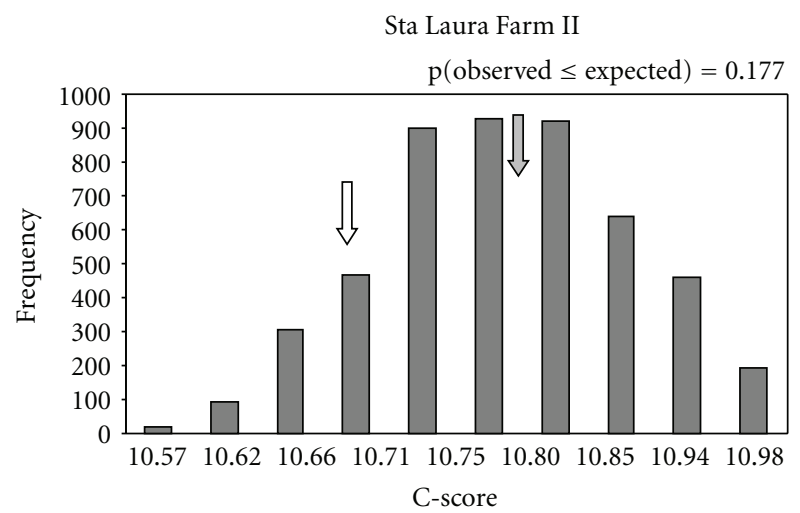

(d)

California Farm

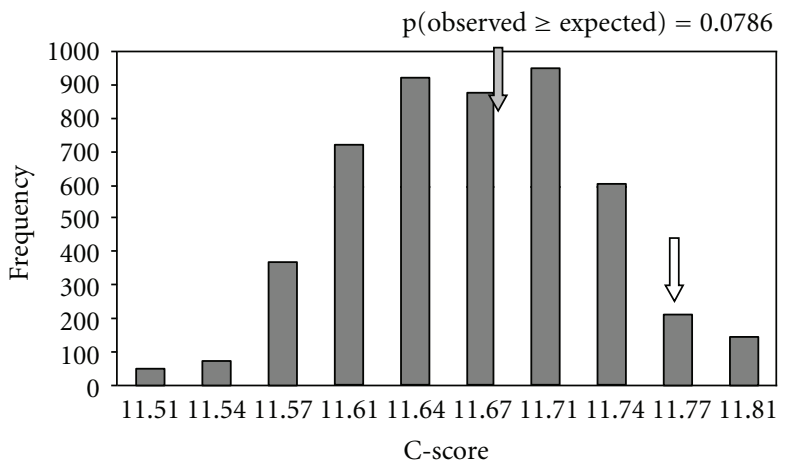

(f)

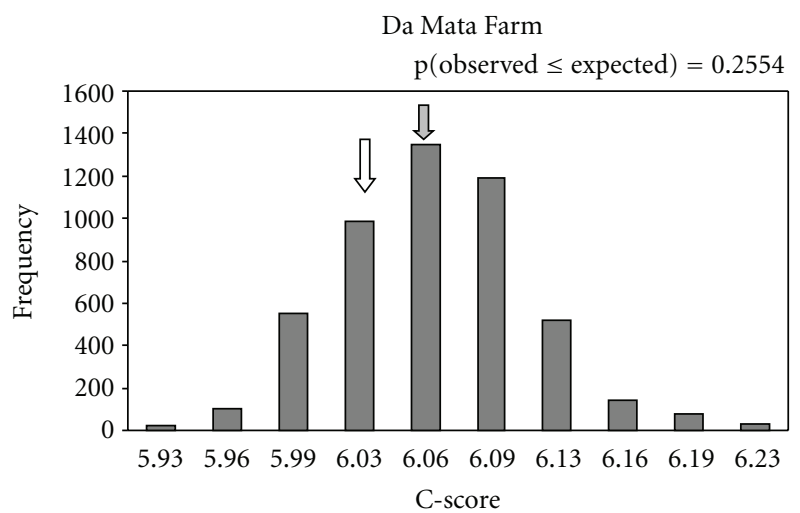

(h)

Figure 8: Continued. 


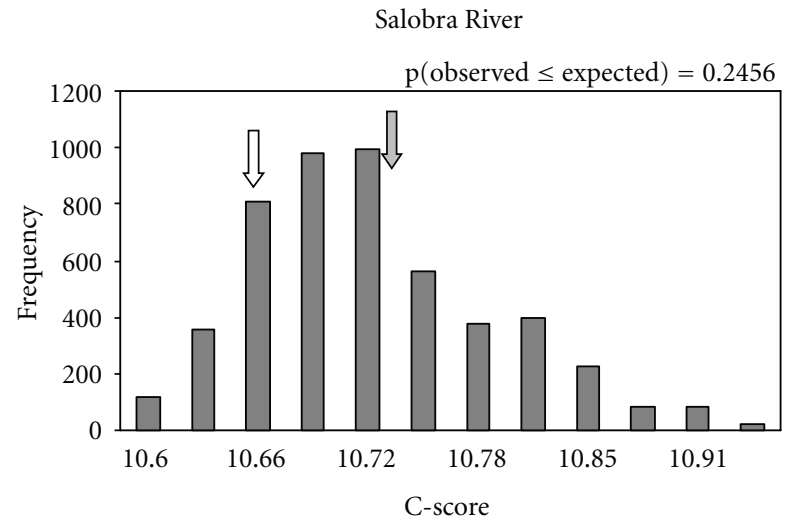

(i)
Sta Maria Farm-Perdido River

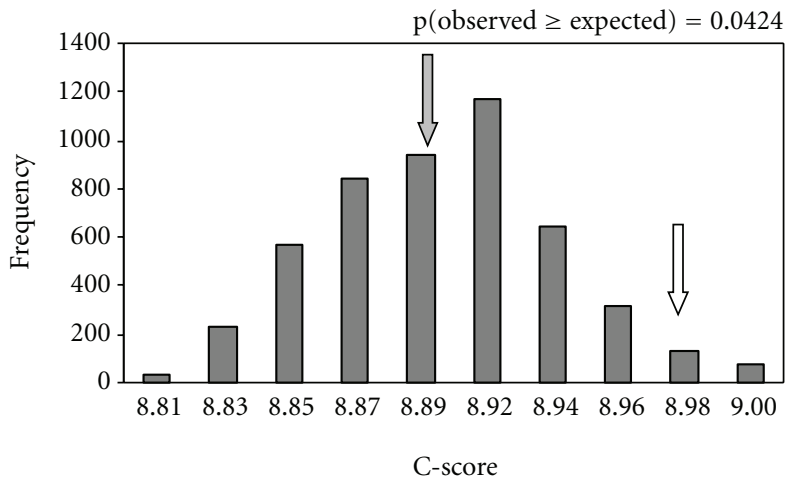

(j)

Figure 8: Co-occurrence analysis comparing 262 Winkler's samples of leaf-litter ants in Serra da Bodoquena. (Full arrow represents the expected values, and empty arrow represents the observed values for C-score measures.)

strongly associated with latitude, elevation, light availability, and vegetation composition, are important at regional spatial scales. Local and regional effects can mask or amplify largerscale latitudinal patterns of species richness.

The differences in species diversity among the study sites could be related to Pleistocene events, such as biota interpenetration between two geologically distinct environments, and the phytophysionomic mosaic occurring in the region. According to Johnson and Ward [48], the topography of an ecotone and adjacent ecosystems is the most important factor affecting ant species richness. Here, each area could similarly allow the entrance of species coming from the surrounding matrix, affecting in turn the distribution of species in the core of the study sites.

There are several possible explanations for the inverse relationship between the observed low alpha diversity and high beta diversity, such as the particular characteristics of the forest fragments. The conservation status and potential connections between forest sites affect species persistence and colonisation in each site. These potential connections have a direct influence on the structure delimited by a buffer (considering the establishment of an influence zone for each area), which leads to an increase in species richness in interconnected forest fragments.

The absence of correlation between similarity and distance suggests that the ant species and assemblages are randomly distributed over the region. The low similarity between consecutive sampled sites suggests a strong formation effect and the influence of adjacent areas, which are evidenced by high beta diversity, with different arrangements of the ant fauna and a high turnover in species dominance across samples.

In forest fragments, ant richness depends on the diversity of local microhabitats and other factors acting at a local scale, such as physical and vegetation structure [33, 49], relief, humidity, and amount of leaf litter available at the location of the food resources and nesting sites used by ants $[50,51]$. Significant variance in species composition can be explained by notable features that shape leaf litter ant communities, namely, litter biomass, soil stoichiometry, heterogeneous distribution of nutrients, soil moisture, invasive species, ecological disturbance at a small scale, and competition dynamics [52-56]. However, it is the ecological and historical biogeography that determines the current composition of the ant assemblages that colonize these micro-habitats (biotic and abiotic filters in the historical evolution of habitats), and also patchiness in space and time which are originated from different sources.

Our results suggest that estimated richness (Chao $2=231.7$, Jackknife $2=250.4$ ) is highly affected by the number of species that were only recorded once ("rare species" $=37$ ). This pattern is in agreement with other studies in the Neotropical Region [5, 32, 57], which have found a high incidence of rare species in ant communities.

The Kadiwéu indigenous reserve, bordering the Pantanal plain, was the locality with lowest similarity conjunct dataset. The deciduous forest in this area forms an enclave of vegetation, influenced by the transition to Cerrado in this area. Transition zones are located at the boundaries between biogeographic regions and represent areas of biotic overlap, which are promoted by historical and ecological changes that allow the mixture of different biotic elements $[9,10]$.

The pattern observed suggests that the structure of the local community is directly affected by the landscape matrix in each region and that it is in fact an ecotone of the Chaco, Cerrado, Atlantic Forest, Amazonian Forest, and Pantanal.

The co-occurrence analysis of leaf-litter ant species indicates that competitive interactions are not the only factors responsible for organising ant assemblages. There is no reason to reject the null hypothesis that the number of checkerboard pairs in the samples is random.

Our results corroborate Andersen [16], agreeing that species coexistence is determined to a significant extent by processes operating during the colonization phase, rather than just by interactions between established colonies, and that competitive outcomes are highly conditioned by 
environmental variation, which severely limits competitive exclusion.

In spite of changes in the extant ant species along a latitudinal gradient in the Cerrado biome [17], community functionality remains similar; this suggests a similar evolutionary ecological history in response to this matrix. In cases where the functionality of a community is distinct, we can assume that the evolutionary history of colonisation came from particular processes and not from a common process (monophyletic). Regarding the functional structure of the community, we suggest that further studies should investigate whether the same guilds are found in the northern and southern portions of Serra da Bodoquena.

\section{Acknowledgments}

The authors thank the members of the "Exército de Libertação da Natureza", our research group from the Hymenoptera Ecology Laboratory (Hecolab/UFGD); Adílio A. Vadadão de Miranda, Fernando Correia Villela and Ivan Salzo "Instituto Chico Mendes de Biodiversidade" in Bonito, Mato Grosso do Sul state; they thank Kadiwéu people to permit them the access to their land. they are also grateful to Jonathan Majer, Carlos Roberto Brandão, Wedson Desidério Fernandes, Rogério Rosa da Silva, Rodrigo Feitosa, Joelson Gonçalves Pereira, Yzel R. Súarez, Sébastien Lacau, Benoît Jahyny, Sarah Groc, and Wesley DaRocha for assistance. This study was carried out under a collection permit from IBAMA (number 10674-11/09/2007). We would also like to thank the Fundação de Apoio ao Desenvolvimento do Ensino, Ciência e Tecnologia do Mato Grosso do Sul-FUNDECT for granting the second author an MSc scholarship (process 41/100.270/2006). JHCD acknowledges his research grant from $\mathrm{CNPq}$.

\section{References}

[1] E. O. Wilson, "A global biodiversity map," Science, vol. 289, no. 5488, p. 2279, 2000.

[2] D. Agosti and L. E. Alonso, "The ALL protocol: a standard protocol for the collection of ground-dwelling ants," in Standard Methods for Measuring and Monitoring Biodiversity, D. Agosti, J. D. Majer, L. E. Alonso, and T. R. Schultz, Eds., pp. 204-206, Smithsonian Institution, Washington, DC, USA, 2000.

[3] L. Lach, C. Parr, and K. Abbott, Ant Ecology, Oxford University Press, Oxford, UK, 2009.

[4] D. H. Wall and J. C. Moore, "Interactions underground," BioScience, vol. 49, no. 2, pp. 109-117, 1999.

[5] R. R. Da Silva and R. Silvestre, "Riqueza da fauna de formigas (Hymenoptera: Formicidae) que habita as camadas superficiais do solo em Seara, Santa Catarina," Papeis Avulsos de Zoologia, vol. 44, pp. 1-11, 2004.

[6] R. R. Silva and C. R. F. Brandão, "Morphological patterns and community organization in leaf-litter ant assemblages," Ecological Monographs, vol. 80, no. 1, pp. 107-124, 2010.

[7] L. E. Alonso, "Ants as indicators of diversity," in Standard Methods for Measuring and Monitoring Biodiversity, D. Agosti, J. D. Majer, L. E. Alonso, and T. R. Schultz, Eds., pp. 80-88, Smithsonian Institution, Washington, DC, USA, 2000.
[8] H. M. André, X. Ducarme, and P. Lebrun, "Soil biodiversity: myth, reality or conning?” Oikos, vol. 96, no. 1, pp. 3-24, 2002.

[9] J. J. Morrone, "Biogeographic areas and transition zones of Latin America and the Caribbean Islands based on panbiogeographic and cladistic analyses of the entomofauna," Annual Review of Entomology, vol. 51, pp. 467-494, 2006.

[10] J. J. Morrone, "What is the Chacoan subregion?" Neotropica, vol. 46, pp. 51-68, 2000.

[11] D. E. Prado and P. E. Gibbs, "Patterns of species distributions in the dry seasonal Forest of South America," Annual Missouri Botanical Garden, vol. 80, pp. 902-927, 1993.

[12] R. Toby Pennington, D. E. Prado, and C. A. Pendry, "Neotropical seasonally dry forests and Quaternary vegetation changes," Journal of Biogeography, vol. 27, no. 2, pp. 261-273, 2000.

[13] J. M. Felfili, "Fragmentos florestais estacionais do Brasil Central: diagnóstico e proposta de corredores ecológicos," in Fragmentação Florestal e Alternativas Para o Desenvolvimento Rural na Região Centro-Oeste, R. B. Costa, Ed., pp. 139-160, UCDB, Campo Grande, MS, Brasil, 2003.

[14] A. Pott and V. J. Pott, "Espécies de fragmentos florestais em Mato Grosso do Sul," in Fragmentação Florestal e Alternativas Para o Desenvolvimento Rural na Região Centro-Oeste, R. B. Costa, Ed., pp. 26-52, UCDB, Grande, MS, Brasil, 2003.

[15] E. Weiher, G. D. P. Clarke, and P. A. Keddy, "Community assembly rules, morphological dispersion, and the coexistence of plant species," Oikos, vol. 81, no. 2, pp. 309-322, 1998.

[16] A. N. Andersen, "Not enough niches: non-equilibrial processes promoting species coexistence in diverse ant communities," Austral Ecology, vol. 33, no. 2, pp. 211-220, 2008.

[17] R. Silvestre, C. R. F. Brandão, and R. R. Da Silva, "Grupos funcionales de hormigas: el caso de los gremios del Cerrado, Brasil," in Introdución a Las Hormigas de la Región Neotropical, F. Fernández, Ed., pp. 113-143, Instituto Humboldt, Bogotá, Colômbia, 2003.

[18] W. J. Stubbs and J. B. Wilson, "Evidence for limiting similarity in a sand dune community," Journal of Ecology, vol. 92, no. 4, pp. 557-567, 2004.

[19] MMA- Ministério do Meio Ambiente Brasil, "Biodiversidade Brasileira: Avaliação e identificação de ações prioritárias para a conservação, utilização sustentável e repartição dos benefícios da biodiversidade brasileira," Brasília, Secretaria de Biodiversidade e Florestas. 2007 http://www.mma.gov.br.

[20] P. C. Boggiani, T. R. Fairchild, and A. M. Coimbra, "O grupo corumbá (Neoproterozóico - Cambriano) na região Central da Serra da Bodoquena, Mato Grosso do Sul (Faixa Paraguai)," Revista Brasileira de Geociências, vol. 23, no. 3, pp. 301-305, 1993.

[21] T. Piacentini, P. C. Boggiani, J. K. Yamamoto, B. T. Freitas, and G. A. C. Campanha, "Formação ferrífera associada à sedimentação glaciogênica da Formação Puga (Marinoano) na Serra da Bodoquena, MS," Revista Brasileira de Geociências, vol. 37, pp. 530-541, 2007.

[22] M. Uetanabaro, F. L. Souza, P. Landgref Filho, A. F. Beda, and R. A. Brandão, "Anfíbios e répteis do Parque Nacional da Serra da Bodoquena, Mato Grosso do Sul, Brasil," Biota Neotropica, vol. 7, no. 3, pp. 279-289, 2007.

[23] B. T. Bestelmeyer, D. Agosti, L. E. Alonso et al., "Field techniques for the study of ground-dwelling ants," in Standard Methods for Measuring and Monitoring Biodiversity, D. Agosti, J. D. Majer, L. E. Alonso, and T. R. Schultz, Eds., pp. 122-144, Smithsonian Institution, Washington, DC, USA, 2000. 
[24] B. Bolton, Identification Guide to the Ant Genera of the World, Harvard University Press, Cambridge, Mass, USA, 1994.

[25] B. Bolton, "Synopsis and classification of formicidae," Memoirs of the American Entomological Institute, vol. 71, pp. 1-374, 2003.

[26] F. Fernández, Introducción a Las Hormigas de la Región Neotropical, Instituto de Investigación de Recursos Biológicos “Alexander von Humboldt", Bogotá, Colombia, 2003.

[27] C. Baroni Urbani and M. L. de Andrade, "The ant tribe Dacetini: limits and constituent genera, with descriptions of new species," Annali del Museo Civico di Storia Naturale "Giacomo Doria”, vol. 99, pp. 1-192, 2007.

[28] J. S. Lapolla, S. G. Brady, and S. O. Shattuck, "Phylogeny and taxonomy of the Prenolepis genus-group of ants (Hymenoptera: Formicidae)," Systematic Entomology, vol. 35, no. 1, pp. 118-131, 2010.

[29] J. T. Longino, J. Coddington, and R. K. Colwell, "The ant fauna of a tropical rain forest: estimating species richness three different ways," Ecology, vol. 83, no. 3, pp. 689-702, 2002.

[30] R. K. Colwell, "EstimateS: statistical estimation of species richness and shared species from samples," Version 7.5. User's Guide and application, 2005, http://purl.oclc.org/estimates.

[31] M. Leponce, L. Theunis, J. H. C. Delabie, and Y. Roisin, "Scale dependence of diversity measures in a leaf-litter ant assemblage," Ecography, vol. 27, no. 2, pp. 253-267, 2004.

[32] R. R. Silva, R. S. M. Feitosa, and F. Eberhardt, "Reduced ant diversity along a habitat regeneration gradient in the southern Brazilian Atlantic Forest," Forest Ecology and Management, vol. 240, no. 1-3, pp. 61-69, 2007.

[33] S. Groc, J. Orivel, A. Dejean et al., "Baseline study of the leaf-litter ant fauna in a French guianese forest," Insect Conservation and Diversity, vol. 2, no. 3, pp. 183-193, 2009.

[34] R. K. Colwell, X. M. Chang, and J. Chang, "Interpolating, extrapolating, and comparing incidence-based species accumulation curves," Ecology, vol. 85, no. 10, pp. 2717-2727, 2004.

[35] R. L. Chazdon, R. K. Colwell, J. S. Denslow, and M. R. Guariguata, "Statistical methods for estimating species richness of woody regeneration in primary and secondary rain forests of NE Costa Rica," in Forest Biodiversity Research, Monitoring and Modeling: Conceptual Background and Old World Case Studies, F. Dallmeier and J. A. Comiskey, Eds., pp. 285-309, Parthenon Publishing, Paris, France, 1998.

[36] P. Legendre and L. Legendre, Numerical Ecology, Elsevier, Amsterdam, The Netherlands, 2nd edition, 1998.

[37] P. H. A. Sneath and R. R. Sokal, Numerical Taxonomy, W. H. Freeman, San Francisco, Calif, USA, 1973.

[38] W. L. Kovach, MVSP-A Multivariate Statistical Package for Windows ver. 3.1, Kovach Computing Services, Pentraeth, Wales, UK, 2005.

[39] N. Mantel, "The detection of disease clustering and a generalized regression approach," Cancer Research, vol. 27, no. 2, pp. 133-153, 1967.

[40] R Development Core Team, R: A Language and Environment for Statistical Computing, R Foundation for Statistical Computing, Viena, Austria, 2009.

[41] J. Oksanen, R. Kindt, P. Legendre, and B. O’Hara, "Vegan: community ecology package," Version 1.8.2, 2005, http:// cc.oulu.fi/ jarioksa.

[42] StatSoft, Inc., "Statistica (data analysis software system), version 6," 2001, www.statsoft.com.
[43] B. D. Coleman, M. A. Mares, M. R. Willig, and Y. H. Hsieh, "Randomness, area, and species richness," Ecology, vol. 63, no. 4, pp. 1121-1133, 1982.

[44] A. E. Magurran, Ecological Diversity and Its Measurement, Princeton University Press, Princeton, NJ, USA, 1988.

[45] N. J. Gotelli and G. L. Entsminger, "EcoSim: null models software for ecology, version 7," Acquired Intelligence Inc. and Kesey-Bear. Jericho, VT 05465, 2011, http://garyentsm inger.com/ecosim/index.htm.

[46] N. J. Gotelli, "Null model analysis of species co-occurrence patterns," Ecology, vol. 81, no. 9, pp. 2606-2621, 2000.

[47] N. J. Gotelli and A. M. Ellison, "Biogeography at a regional scale: determinants of ant species density in New England bogs and forests," Ecology, vol. 83, no. 6, pp. 1604-1609, 2002.

[48] R. A. Johnson and P. S. Ward, "Biogeography and endemism of ants (Hymenoptera: Formicidae) in Baja California, Mexico: a first overview," Journal of Biogeography, vol. 29, no. 8, pp. 1009-1026, 2002.

[49] S. A. Lassau and D. F. Hochuli, "Effects of habitat complexity on ant assemblages," Ecography, vol. 27, no. 2, pp. 157-164, 2004.

[50] C. A. Brühl, G. Gunsalam, and K. E. Linsenmair, "Stratification of ants (Hymenoptera, Formicidae) in a primary rain forest in Sabah, Borneo," Journal of Tropical Ecology, vol. 14, no. 3, pp. 285-297, 1998.

[51] N. L. Hites, M. A. N. Mourão, F. O. Araújo, M. V. C. Melo, J. C. De Biseau, and Y. Quinet, "Diversity of the ground-dwelling ant fauna (Hymenoptera: Formicidae) of a moist, montane forest of the semi-arid Brazilian "Nordeste", Revista de Biologia Tropical, vol. 53, no. 1-2, pp. 165-173, 2005.

[52] S. M. Alvarenga, A. E. Brasil, and D. M. Del Arco, "Projeto RADAM/BRASIL," Geomorfologia, vol. 28, pp. 125-138, 1982.

[53] A. York, "Long-term effects of frequent low-intensity burning on ant communities in coastal blackbutt forests of southeastern Australia," Austral Ecology, vol. 25, no. 1, pp. 83-98, 2000.

[54] T. P. McGlynn, R. M. Fawcett, and D. A. Clark, "Litter aiomass and nutrient determinants of ant density, nest size, and growth in a Costa Rican tropical wet forest," Biotropica, vol. 41, no. 2, pp. 234-240, 2009.

[55] F. B. Baccaro, S. M. Ketelhut, and J. W. De Morais, "Resource distribution and soil moisture content can regulate bait control in an ant assemblage in Central Amazonian forest," Austral Ecology, vol. 35, no. 3, pp. 274-281, 2010.

[56] A. E. Dunham and A. S. Mikheyev, "Influence of an invasive ant on grazing and detrital communities and nutrient fluxes in a tropical forest," Diversity and Distributions, vol. 16, no. 1, pp. 33-42, 2010.

[57] J. H. C. Delabie, D. Agosti, and I. C. Nascimento, "Litter ant communities of the Brazilian Atlantic rain forest region," Curtin University School of Environmental Biology Bulletin, vol. 18, pp. 1-17, 2000. 

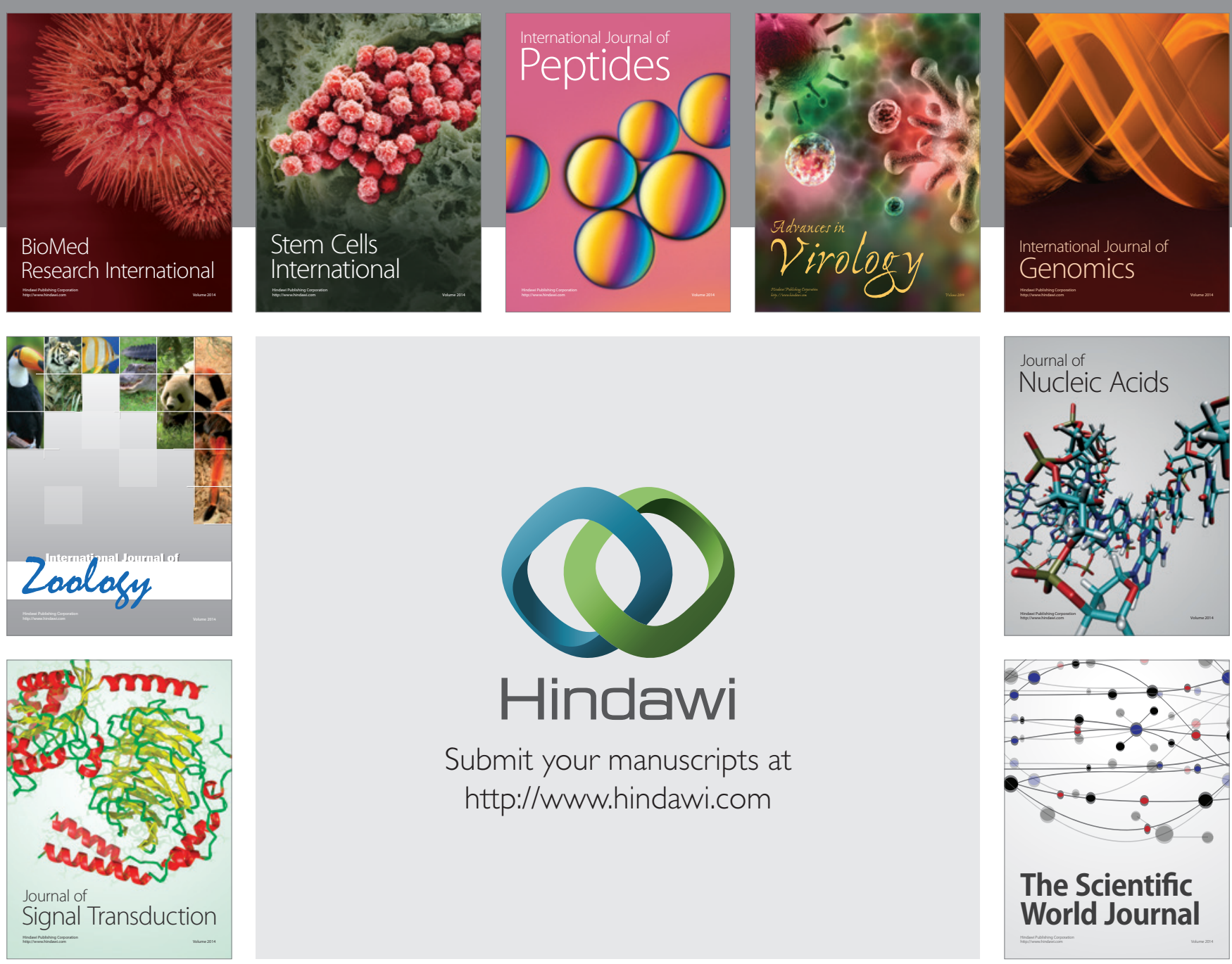

Submit your manuscripts at

http://www.hindawi.com
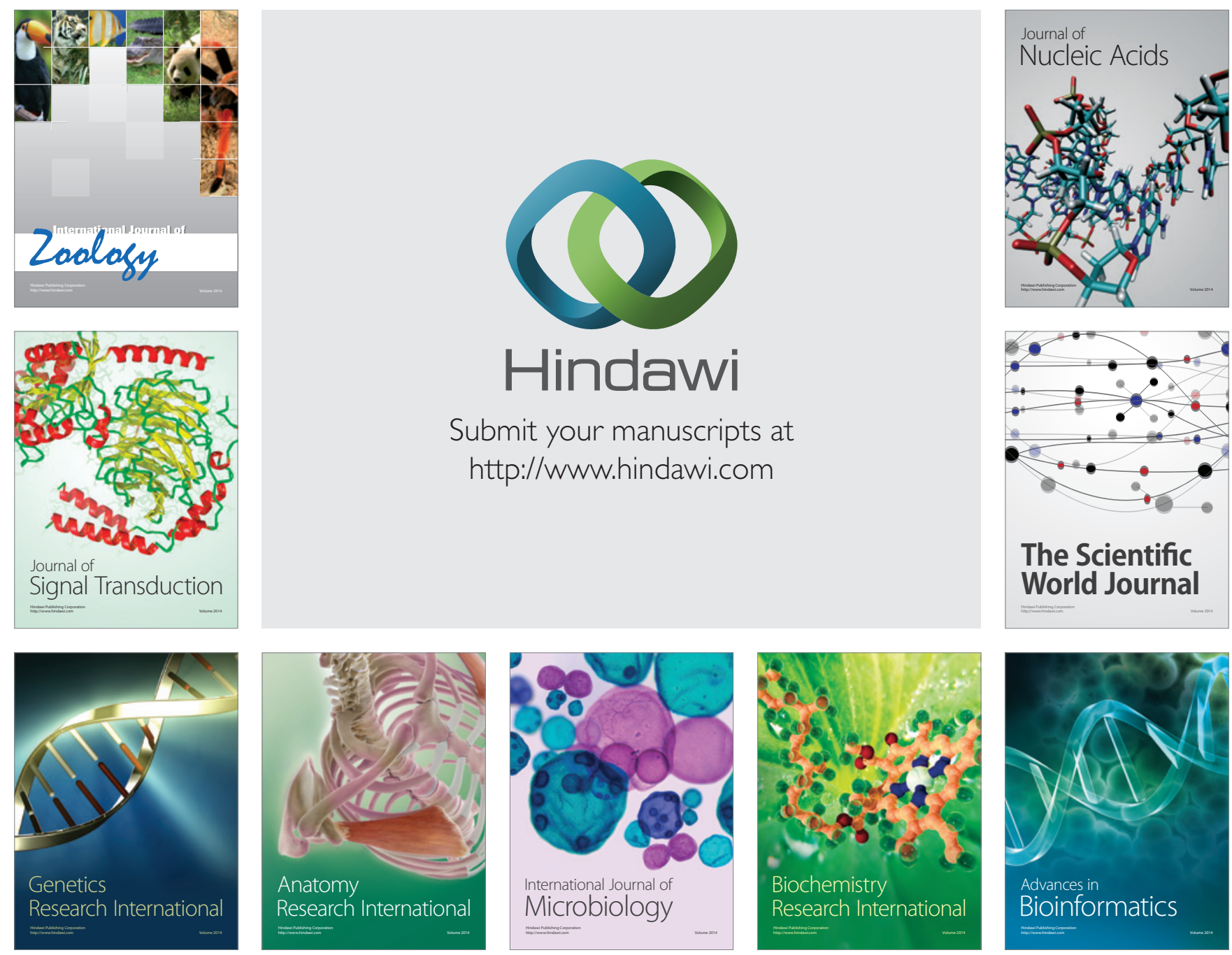

The Scientific World Journal
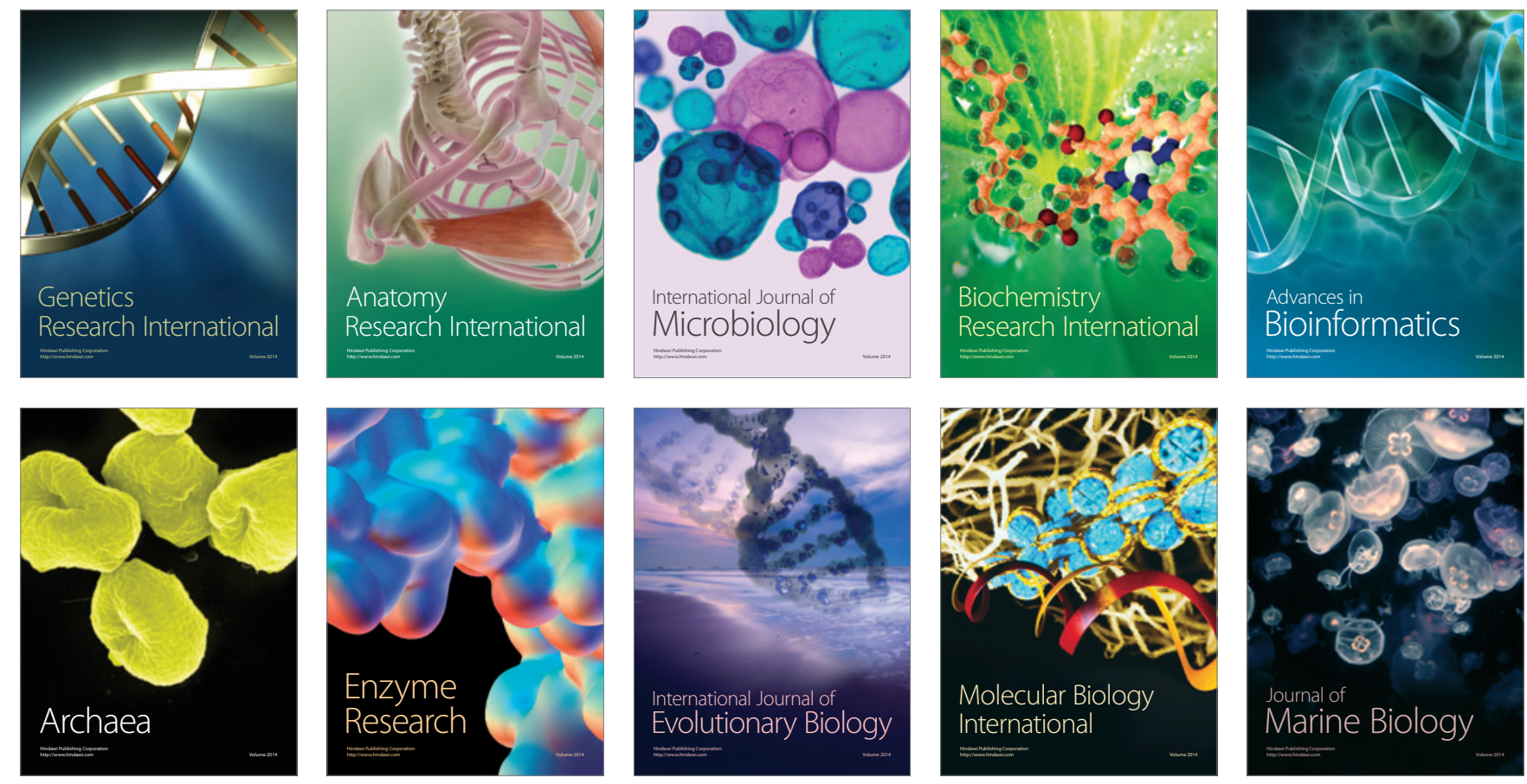